\title{
Embedded Kinetic Simulation of Ganymede's Magnetosphere: Improvements and Inferences
}

\author{
Hongyang Zhou ${ }^{1}$, Gábor Tóth ${ }^{1}$, Xianzhe Jia ${ }^{1}$, Yuxi Chen ${ }^{1}$, Stefano Markidis ${ }^{2}$ \\ ${ }^{1}$ Department of Climate and Space Sciences and Engineering, University of Michigan, Ann Arbor, \\ Michigan, USA \\ ${ }^{2}$ Department for Computational Science and Technology, KTH Royal Institute of Technology, Stockholm, \\ Sweden
}

\section{Key Points:}

- Improved Hall MHD-EPIC model of Ganymede's magnetosphere with realistic inner boundary conditions and energy-conserving PIC scheme.

- Fully resolved ion-scale kinetics at upstream magnetopause, shown by the nongyrotropic/anisotropic distributions.

- Electron precipitation of $\sim 10^{-7} \mathrm{~W} / \mathrm{cm}^{2}$ shows up to half of the peak emission brightness contributed by upstream reconnection.

This is the author manuscript accepted for publication and has undergone full peer review but has not been through the copyediting, typesetting, pagination and proofreading process, which may lead to differences between this version and the Version of Record. Please cite this article as doi: 10.1029/2019JA026643

Corresponding author: Hongyang Zhou, hyzhou@umich.edu

This article is protected by copyright. All rights reserved. 


\begin{abstract}
The largest moon in the solar system, Ganymede, is also the only moon known to possess a strong intrinsic magnetic field and a corresponding magnetosphere. Using the new version of Hall MHD-EPIC model with a self-consistently coupled resistive body representing the electrical properties of the moon's interior, improved inner boundary conditions, and the flexibility of coupling different grid geometries, we achieve better match of magnetic field with measurements for all six Galileo flybys. The G2 flyby comparisons of plasma bulk flow velocities with the PLS data support the oxygen ion assumption inside Ganymede's magnetosphere. Crescent shape, nongyrotropic and non-isotropic ion distributions are identified from the coupled model. Furthermore, we have derived the energy fluxes associated with the upstream magnetopause reconnection of $\sim 10^{-7} \mathrm{~W} / \mathrm{cm}^{2}$ based on our model results and found a maximum of $40 \%$ contribution to the total peak auroral emissions.
\end{abstract}

\title{
1 Introduction
}

Ganymede, the largest satellite in our solar system, is of great interest to magnetosphere research because it is the only known satellite to be strongly magnetized (M. Kivelson et al., 1996). The dynamics happening in its mini-magnetosphere from sub-Alfvénic interaction between Jovian plasma and the moon's intrinsic field offers a unique example of comparative studies to other plasma interaction systems. The typical flow speed of the ambient plasma relative to Ganymede is less than the magnetosonic speed, so there is no bow shock forming upstream of the moon; instead, the interaction of the magnetosphere with the sub-Alfvénic flow forms an Alfvén wing structure.

In the past decades, tremendous effort and progress have been made in the study of the plasma interaction and dynamic processes happening in Ganymede's magnetosphere. The global structure of Ganymede's magnetosphere as a consequence of sub-Alfvénic plasma interaction is described in depth by M. G. Kivelson et al. (2004). Starting from ideal MHD models and simulations, solid basics in understanding the Alfvén wing structure and fundamental plasma convections have been established. Dorelli et al. (2015) stressed the importance of the Hall effect in a global magnetosphere model. The decoupling of ion and electron motion not only introduces Hall magnetic field near the reconnection site, but also distorts the magnetosphere's global convection pattern dramatically compared to ideal/resistive MHD. Even though previous simulation studies of Ganymede's magnetosphere environment with single-fluid ideal MHD (Jia et al., 2008, 2009, 2010; Duling et al., 2014), multi-fluid ideal MHD (Paty \& Winglee, 2004, 2006; Paty et al., 2008), Hall MHD (Dorelli et al., 2015), and hybrid model (Fatemi et al., 2016) have achieved good agreement with observations, these models did not provide a fully kinetic description of Ganymede's magnetosphere that is expected to play an important role in magnetic reconnection.

Multi-scale physics is one of the primary concerns in global plasma simulations. Studies of magnetopause reconnection have gone through MHD scales to kinetic ion and electron scales (Eastwood et al., 2016; Burch et al., 2016), providing a more detailed microscopic view of the energy transfer processes within the magnetosphere. Due to the fact that ion inertial length $d_{i} \sim 425 \mathrm{~km} \sim 0.16 R_{G}$ and ion gyroradius $r_{i} \sim 200 \mathrm{~km}$ are quite large near Ganymede's magnetopause (Neubauer, 1998), the ion (and possibly electron) kinetic effects at Ganymede's magnetosphere are expected to be important. The small size of Ganymede's magnetosphere provides a great opportunity to employ the magnetohydrodynamic with embedded particle-in-cell (MHD-EPIC) model (Daldorff et al., 2014) to capture kinetic effects in a global model. The current computational capability allows us to fully resolve the ion scale near Ganymede and study reconnection in a more kinetic way. Tóth et al. (2016) described the first global MHD-EPIC model for Ganymede's magnetosphere in detail. This coupling model has also been successfully applied to part 
of other plantery magnetospheres like the magnetotail of Mars (Ma et al., 2018) and Earth's dayside magnetopause (Chen et al., 2017).

Inspired by previous ideal MHD simulations on a stretched spherical grid with a coupled resistive interior of the moon (Jia et al., 2009), here we combine the MHD-EPIC approach with the resistive body model by using a stretched spherical MHD grid and a Cartesian PIC grid. With the improved fluid-kinetic coupling model, we are able to gain insights into the global effects of kinetic physics in time-dependent simulations and study the influence of upstream conditions on the global structure as well as dynamics of Ganymede. Compared with our previous work, our new simulation model presented in this paper includes the following major improvements: 1 . the total energy inside the PIC box region is conserved; 2. assumption of fixed ratio between electron and ion pressures (except at boundaries) in the MHD model Tóth et al. (2016) is being removed, and; 3. the inner boundary description has been improved using a resistive body approach, similar to that used by Jia et al. (2009) in simulating Ganymede's magnetosphere. We extended our simulation to all the available Galileo flybys and ran the two upstream flybys G8 and G28 simulations for 20 minutes of physical time to cover the whole magnetosphere crossing.

All the input parameters used in our simulations are inferred from the observations by the Galileo spacecraft that recorded six close encounters of Ganymede during its eight years in the Jovian system. The G1, G2, G7 and G29 flybys went across the Alfvén wing near the north pole in the slightly downstream region, while G8 and G28 flybys went through the open-closed field line boundary at low latitudes on the upstream side. The latter two in-situ measurements are of particular interest for studying the upstream magnetosphere, which is the focus of this paper. During the G8 flyby, the close encounter that occurred near the central plasma sheet of Jupiter, Galileo detected what appeared to be large-amplitude waves in the magnetic field at the magnetopause crossings (see Figure 6d). The fluctuations were present both at the entry and exit of the magnetosphere. These signatures have been suggested by Jia et al. (2010) to be closely related to magnetic reconnection and flux transfer events (FTEs) (Southwood et al., 1988; Russell \& Elphic, 1978) at the magnetopause which we can diagnose from simulated 3D outputs. Tóth et al. (2016) have confirmed that these are FTEs indeed. We show that with the improved MHD-EPIC model we are able to capture not only part of the transient signatures observed by Galileo, but also the global picture of the plasma interaction system. Detailed ion phase space distributions are presented near the upstream reconnection region, and energetic flux densities from the upstream magnetopause are obtained and mapped to the surface, showing the influence of reconnection on the ion/electron precipitation. The resulting auroral emission is estimated and compared with observations.

This paper is organized as follows: the models are described in section 2, the results are presented in section 3 followed with discussions in section 4 , and an overall summary in section 5 .

\section{Methods}

The simulations presented in this paper are performed with the space weather modeling framework (SWMF) (Tóth et al., 2012). The global structure is simulated by a HallMHD model, with an embedded PIC region at the upstream magnetopause. The MHD and PIC models exchange information for plasma and electro-magnetic field through the framework. As an extension to the previous MHD-EPIC modeling work on Ganymede (Tóth et al., 2016), we now solve the Hall MHD equations with a separate electron pressure equation in BAT-S-RUS (Powell et al., 1999; Tóth et al., 2008). The magnetic induction equation is solved throughout the mantle of the moon to allow for the magnetic field to diffuse through the planetary body. Electrons and ions inside the upstream reconnection region covered by the PIC box are simulated with a semi-implicit energy-conserving 
scheme (Lapenta, 2017) that was further improved by Chen and Toth (2018) and implemented into iPIC3D (Markidis et al., 2010).

\subsection{Hall MHD Model: BAT-S-RUS}

The Hall MHD code is described in Tóth et al. (2008), and here we briefly summarize and present the run parameters. For all the simulations of Ganymede's magnetosphere, we choose an explicit-implicit timestepping scheme and a numerical flux of Sokolov scheme Sokolov et al. (1999) with third-order monotonized central (Koren) limiter in BATS-RUS. The Hall effect is restricted to $|x|<5 R_{G},|y|<4 R_{G}$ and $|z|<4 R_{G}$ box region excluding a sphere of radius $1.05 R_{G}$ centered at the moon to speed up the simulation. $(x, y, z)$ is defined in the GphiO system, where $\widehat{x}$ is along the flow direction, $\widehat{y}$ is along the Ganymede-Jupiter vector with positive direction pointing towards Jupiter, and $\widehat{z}$ is along the spin axis.

The Hall MHD equations (with electron pressure gradient term and separate electron pressure equation) to be solved are

$$
\begin{aligned}
\frac{\partial \rho}{\partial t} & =-\nabla \cdot(\rho \mathbf{u}) \\
\frac{\partial(\rho \mathbf{u})}{\partial t} & =-\nabla \cdot\left(\rho \mathbf{u u}+\left(p+p_{e}\right) \overline{\bar{I}}+\frac{B^{2}}{2 \mu_{0}} \overline{\bar{I}}-\frac{\mathbf{B B}}{\mu_{0}}\right) \\
\frac{\partial e}{\partial t} & =-\nabla \cdot\left[(\epsilon+p) \mathbf{u}+\left(\epsilon_{e}+p_{e}\right) \mathbf{u}_{e}+\mathbf{u}_{e} \cdot\left(\frac{\mathbf{B}^{2}}{\mu_{0}} \overline{\bar{I}}-\frac{\mathbf{B B}}{\mu_{0}}\right)-\mathbf{B} \times \eta \mathbf{j}\right],(3) \\
\frac{\partial \mathbf{B}}{\partial t} & =-\nabla \times\left[\mathbf{u}_{e} \times \mathbf{B}+\eta \mathbf{j}+\frac{\nabla p_{e}}{n e}\right] \\
\frac{\partial p_{e}}{\partial t}+\nabla \cdot\left(p_{e} \mathbf{u}_{e}\right) & =-(\gamma-1) p_{e} \nabla \cdot \mathbf{u}_{e} .
\end{aligned}
$$

where $\overline{\bar{I}}$ is the identity matrix, $\rho$ is the mass density, $\mathbf{u}$ is the plasma bulk velocity, B is the magnetic field, $p_{e}$ is the electron pressure, $p$ is the ion thermal pressure, and $\mathbf{j}=$ $\nabla \times \mathbf{B} / \mu_{0}$ is the current density. The Hall velocity is defined as

$$
\mathbf{v}_{H}=-\frac{\mathbf{j}}{n e},
$$

and the electron bulk velocity is given by

$$
\mathbf{u}_{e}=\mathbf{u}+\mathbf{v}_{H} .
$$

The total energy density is

$$
e=\epsilon+\epsilon_{e}+\frac{B^{2}}{2 \mu_{0}}=\frac{1}{2} \rho \mathbf{u}^{2}+\frac{1}{\gamma-1}\left(p+p_{e}\right)+\frac{\mathbf{B}^{2}}{2 \mu_{0}},
$$

where $\epsilon$ is the hydrodynamic energy density and $\gamma$ is the adiabatic index. Note that in our Hall MHD model only $\left(\rho, \mathbf{u}, \mathbf{B}, p, p_{e}\right)$ are unknowns; all others are derived quantities.

We use a stretched spherical grid in GphiO coordinate system with adaptive mesh refinement (AMR) up to 2 levels near Ganymede's magnetosphere and Alfvén wing structures, enabling high resolution in $r$ about $0.02 R_{G}(\sim 50 \mathrm{~km}), \theta$ of $0.7^{\circ}$ and $\phi$ of $1.4^{\circ}$ near the moon's surface. In order to include Ganymede's Alfvén wing structure as much as possible and avoid the unphysical wave reflection issue at the outer boundaries, we set a large simulation domain of a cube centered at the moon with edge length $l=200 R_{G}$ cut out of the spherical grid, as shown in Figure 1(a). The grid near the inner boundary from $r=0.5 R_{G}$ to $r=5 R_{G}$ is shown in Figure 1(b). 
The continuity equation (1), momentum equation (2), energy equation (3) and electron pressure equation (5) are solved with an explicit timestepping scheme, while the magnetic induction equation (4) is solved with a semi-implicit scheme. Specifically, the convection term $\mathbf{u} \times \mathbf{B}$ and electron pressure gradient term $\nabla p_{e} /$ ne are advanced using explicit timestepping, while the resistivity term $\eta \mathbf{J}$ and Hall term $\mathbf{v}_{H} \times \mathbf{B}$ are advanced with an implicit scheme to handle the stiff problems without limiting the discrete timesteps.

The hyperbolic cleaning and eight-wave schemes (Dedner et al., 2002; Powell et al., 1999) are used to keep the divergence-free magnetic field constraint. Special treatment of small cells near the axis of symmetry of the spherical grid is taken into account by smoothing the cell-center quantities in $\widehat{\phi}$ to increase the timesteps and reduce the discontinuities near the pole axis. In addition, an accurate parallel fieldline-tracing scheme is used to capture the open-closed field line boundary and magnetic field topology.

\subsection{Particle-in-Cell Model: iPIC3D}

The particle-in-cell (PIC) code iPIC3D uses a semi-implicit integration in time of the Vlasov-Maxwell system, which removes the numerical stability constraints encountered in explicit time-stepping and enables kinetic plasma simulations at magnetohydrodynamic time scales (Markidis et al., 2010). In Cartesian coordinates, we set a PIC box at the upstream magnetopause between $-2.5 R_{G} \leq x \leq-1.125 R_{G},-2 R_{G} \leq y \leq$ $2 R_{G},-2.2 R_{G} \leq z \leq 2.2 R_{G}$ with grid resolution $1 / 32 R_{G}\left(\sim 0.2 \lambda_{i}\right.$, or $\left.2 \lambda_{e}\right)$. The speed of light $\mathrm{c}$ is reduced to $4000 \mathrm{~km} / \mathrm{s}$ to decrease the number of iterations for the implicit solver, and the ion-electron mass ratio $m_{i} / m_{e}$ is set to 100 to reduce the scale separation of electron skin depth and ion inertial length.

The Energy Conserving Semi-Implicit Method (ECSIM) from Lapenta (2017) has recently been implemented into iPIC3D Chen and Toth (2018), with further improvements including new methods of maintaining Gauss's law $\nabla \cdot \mathbf{E}=\rho / \epsilon_{0}$ by corrections of the particle positions and improved suppression of numerical oscillations. This divergence E cleaning method at each step has been fully adopted into our coupled simulation.

We initialize the PIC code with 216 ions and 216 electrons respectively in each cell with Maxwellian distribution based on the MHD state at the beginning of the time-dependent simulation. Then we run the coupled model, and allow macro-particles to be lost at the boundary or enter the PIC domain as the system evolves. The coupling precedure with MHD is described further below.

\subsection{Initial Conditions}

Ganymede's magnetic field is composed of a permanent dipole generated by the core and an induced dipole from interactions with the time-varying Jovian magnetic field (M. Kivelson et al., 2002). Both dipole moments can be calculated from statistical fitting of Galileo magnetometer data over flybys, while higher order moments are ignored in our simulations. Table 1 lists the total dipole moments for each flyby. The initial conditions for primitive variables are based on the upstream conditions. The plasma density is set to the observation average of $4 \mathrm{~cm}^{-3}$ for G8 flyby where Ganymede's orbit is inside Jupiter's current sheet and $2 \mathrm{~cm}^{-3}$ for other flybys with an average mass per unit charge $14 \mathrm{amu}$ representing a mixture of $\mathrm{H}^{+}$and $\mathrm{O}^{+}$. We assume ions are singly charged and treat energetic and thermal ions as a single fluid. The total thermal pressure of the ambient plasma is set as $p_{t}=3.8 \mathrm{nPa}$ for $\mathrm{G} 8$ flyby, and $p_{t}=1.9 \mathrm{nPa}$ for the other five flybys which occurred away from the central plasma sheet, according to M. G. Kivelson et al. (2004). 


\subsection{Boundary Conditions}

There are three kinds of boundaries in the BAT-S-RUS model: the outer boundary (cut off by a box), the surface boundary $\left(r=1 R_{G}\right)$, and the core boundary $(r=$ $\left.0.5 R_{G}\right)$. Figure 2 shows a sketch of the three boundary geometries.

We are using steady upstream conditions for each flyby simulation since Jupiter's rotation period $(\sim 10 \mathrm{~h})$ and Ganymede's orbital period $(\sim 7.15 \mathrm{~d})$ are much longer than the time it takes for Jupiter's corotating plasma to flow past Ganymede's magnetospheric system, which is on the order of minutes (Jia et al., 2008). For the outer boundaries, we specify primitive variables $\rho, \mathbf{u}, \mathbf{B}, p, p_{e}$ at upstream and downstream faces with constant values corresponding to those observed by Galileo for each individual pass as shown in Table 1. The ion-electron temperature ratio is set to 18 based on Table 21.1 in M. G. Kivelson et al. (2004). We apply a fixed boundary condition at the upstream and downstream faces because the flow the subsonic and sub-Alfvénic. The other sides of the outer box boundary are set with zero-gradient boundary conditions to allow for the Alfvén wings, although fixed boundaries would also work.

For the inner boundaries, we followed Jia et al. (2009) with special care on velocities and magnetic fields. The dipole field in our model is set as the sum of a permanent dipole $\mathbf{B}_{0}$ and a prescribed induced dipole field $\mathbf{B}_{1}$ due to the interaction between the time-varying background field and the conducting layer calculated by M. Kivelson et al. (2002). The dipole moments at the core boundary $0.5 R_{G}$ are shown in Table 1 . In the MHD model we only solve for $\mathbf{B}_{1}$ while keeping $\mathbf{B}_{0}$ constant. The semi-implicit scheme implemented in BAT-S-RUS allows us to solve for $\vec{B}$ contributed from the magnetic diffusion term separately from the convection term. This capability has been successfully applied to model the induction effect at Mercury (Jia et al., 2015), and becomes more robust with the new option of setting multi-boundary layer in BATS-R-US. During each timestep, the surface boundary $\left(r=1 R_{G}\right)$ is turned on at first to solve for $\rho, \mathbf{u}, \mathbf{B}, p, p_{e}$ from surface boundary to the outer boundary. A mass density of $550 \mathrm{amu} / \mathrm{cm}^{3}$ with an average temperature of $20 \mathrm{eV}$ (corresponding to pressure $p=n k_{B} T=0.125 \mathrm{nPa}$ ) are fixed, and the flow velocity is set to be continuous perpendicular to the local magnetic

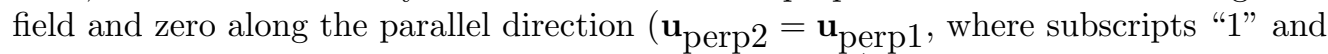
"2" denote the physical and ghost cell faces respectively) at the surface boundary. Then the surface boundary is turned off, and the magnetic diffusion term with resistivity as well as the Hall term are calculated with an implicit scheme and added to $\mathbf{B}$. The inner boundary of the Hall region is set slightly away from the surface at $r=1.05 R_{G}$ to avoid numerical issues. The core boundary $\left(r=0.5 R_{G}\right)$ for $\mathbf{B}$ is always set to a fixed dipole during the flyby. After everything is updated, we move on to the next timestep and repeat the above process. The boundary conditions are summarized in Table 2 .

It has been suggested from both gravity (Anderson et al., 1996) and magnetometer (Schubert et al., 1996) measurements that Ganymede's interior is most likely composed of a metallic core of radius $0.15-0.5 R_{G}$ that sustains the moon's internal magnetic field and a silicate mantle enclosed by an ice shell. This implies that the interior of Ganymede and its ionosphere together with the ambient space plasma have different electrical conductivity. We therefore set an ad hoc resistivity profile in the model shown in Figure 3 as a function of radial distance $r$ (assuming spherical symmetry in $\theta$ and $\phi$ ). To include the effect of the moon's interior, the innermost simulation boundary is placed at $r=0.5 R_{G}$, i.e. the maximum of inferred core radius. Between the core boundary $\left(0.5 R_{G}\right)$ and the moon's surface $\left(1 R_{G}\right)$ is the insulating rocky mantle whose electrical conductivity is extremely low. Note that there is no plasma flowing in this region so the model solves only a diffusion-like equation $\partial \mathbf{B} / \partial t=-\nabla \times(\eta \nabla \times \mathbf{B})$ for the magnetic field. Also as a numerical approximation to a conducting core surface, we set the resistivity to zero at the physical cells next to the core boundary so that the magnetic field does not change and remains equal to the dipole field value. Due to the constraints from geophysical measurements (Anderson et al., 1996; Schubert et al., 1996), we apply a sim- 
plified, spherically symmetric resistivity profile from the core boundary at $r=0.5 R_{G}$ to a radius slightly above the surface $r=1.05 R_{G}$. A surface resistivity $\sim 4 \times 10^{5} \Omega \cdot m$ (within the range of the magnetic diffusivity estimation by Duling et al. (2014)) is used in our simulations, and the overall profile is similar to (Jia et al., 2009). The resistivity profile set from $r=1 R_{G}$ to $r=1.05 R_{G}$ couples the magnetic field inside and outside the moon in our numerical approach and serve as the simplest treatment of ionosphere conductivities. The flowing plasma outside the ionosphere $\left(r>1.05 R_{G}\right)$ is regarded as infinitely conducting, so the background resistivity is set to zero. The extension of the resistive region above the moon's surface is necessary to properly couple the two regions.

\subsection{Coupling Procedure}

We used a stretched spherical grid in the whole computational domain for MHD, and a uniform Cartesian grid for PIC. Interpolation and communication of variables between the two models is handled by the coupler. In time-accurate simulations, BATSR-US and iPIC3D advance with different timesteps individually, and they exchange information every $0.02 s$. The PIC model overwrites the MHD solution in the overlapped region, and obtains time-dependent boundary conditions from the MHD solution. Figures 4 and 5 show the PIC box embedded inside the MHD domain.

Computationally, we go through the steps as follows. First we obtain a quasi-steady state solution by running BATS-R-US in local timestep mode for 30,000 steps in ideal MHD and 20,000 steps in Hall MHD in the full computational domain. Then we continue the simulation in time-accurate mode coupled with iPIC3D covering a box region at the upstream magnetopause. The MHD-EPIC model runs for 20 minutes in physical time and the outputs are saved every second. Taking advantage of a semi-implicit scheme for solving the magnetic induction equation, we are allowed to use time steps that are not limited by whistler waves and the large resistivity in the subsurface ocean and mantle regions. The mixture of explicit-implicit timestepping enables us to construct a robust model with affordable computational cost.

\section{Results}

\subsection{Magnetic Field Comparison With Observation}

The most significant feature in sub-Alfvénic plasma interaction is the Alfvén wing. The global structures including the Alfvén wing and magnetopause near Ganymede are shown in Figure 4 for the G8 flyby $\left(\sim 180^{\circ}\right.$ upstream magnetic field clock angle with respect to the $\mathbf{z}$ axis) and Figure 5 for the G28 flyby ( $\sim 135^{\circ}$ clock angle). Since the intrinsic dipole is only $4^{\circ}$ off from $-\mathbf{z}$ axis (M. Kivelson et al., 2002), these two upstream flybys can represent two typical cases with nearly anti-parallel reconnection and strong guide field reconnection, respectively. In the 3D visualization plots, the red surface represents the open-closed field line boundary, and the yellow surface displays the boundary of field lines with one end connected to the moon and the other end connected to the outer boundaries. During the G8 flyby, the perturbed field lines within the yellow surface form the nearly symmetric Alfvén wing structure over the poles, while for the G28 flyby the southern Alfvén wing is tilted towards the sub-Jovian side and the northern Alfvén wing is tilted towards the anti-Jovian side. At the upstream magnetopause, the topology of magnetic field lines are quite different with/without the guide field (approximately $B_{y}$ component).

With Hall physics included in our simulation, there is no true steady state in the global dynamic system, but we can obtain an approximately steady state solution. First we validate the quasi-steady state Hall MHD solution for all six Galileo flybys. The magnetic field comparisons are shown in Figure 6. The Galileo trajectories for G1, G2, G7 
and G29 flybys go through the Alfvén wing near the north pole region, while the trajectories for G8 and G28 flybys go across the upstream low latitude magnetosphere. Note that PIC is not turned on in the steady state convergence runs. Due to the fact that the conductance of the mantle, subsurface ocean and ionosphere are not well constrained observationally, our choice of conductivity profile is simply based on estimation of reasonable range and comparisons with observed magnetic fields. Our simulation tests show that the magnetic field near the moon is very sensitive to the profile of conductivity as well as the grid resolution and structure. Taking conductivity into consideration in such a small magnetosphere can significantly modify its size and shape. With the inclusion of the resistive body, the overall size of the magnetosphere matches better with observations such that no stretching or shifting of the Galileo trajectory is needed, in contrast with Dorelli et al. (2015) and Tóth et al. (2016) The validation here shows that our Hall MHD model can faithfully reproduce the magnetic field observed by Galileo under different upstream conditions. For the four Alfvén wing flybys, G1, G2, G7 and G29, the differences in the magnetic field data comparisons indicate that the location or size of our simulated Alfvén wing is slightly different from observation. Especially during the inbound crossings of the G7 and G29 flybys, the locations of the Alfvén wing are shifted inward, which is similar to the resistive MHD simulation results by Jia et al. (2009). The agreement in the two upstream magnetopause crossings shows that the position of openclosed field line boundary is well captured in Hall MHD, which gives a nice starting point of incorporating the PIC box region at the upstream magnetosphere.

\subsection{Comparison with Plasma Measurements}

Plasma measurements from the Galileo Plasma Subsystem (PLS) have been published for the G2 flyby (Frank et al., 1997b). Collinson et al. (2018) recently re-analyzed the PLS data to obtain a new set of plasma moments for the G1 and G2 flybys. Here we compare the plasma velocities for the G2 flyby from our Hall-MHD simulation with the PLS moments obtained by both Frank et al. (Figure 9a) and Collinson et al. (Figure $9 \mathrm{~b}$ ). Following the approach used in the same kind of comparison in Jia et al. (2009), we have plotted two velocity profiles for the PLS data corresponding to two different assumptions of the ion mass-per-charge (M/Q): blue and black dots for heavy ions of $M / Q=$ 16, and gray dots for light ions of $\mathrm{M} / \mathrm{Q}=1$. The velocity components shown in Figure 9 have been decomposed into the parallel and perpendicular directions with respect to the magnetic field.

First of all, outside the magnetosphere (the magnetopause crossings are marked by the vertical dashed lines), the flow velocities in our model agree very well with the PLS velocity data derived by Frank et al. (1997b), but deviate from the Collinson et al. (2018) study that gave much reduced plasma speeds. It is important to point out that our simulation assumes a nominal flow speed of $\sim 150 \mathrm{~km} / \mathrm{s}$ for the unperturbed Jovian plasma at the upstream boundary.

Secondly, inside the magnetosphere, due to lack of direct measurement of the ion mass-per-charge, there is ambiguity in deriving the plasma moments using the raw PLS measurements. Therefore, the previously published work typically provide two sets of moments with different assumptions of ion mass-per-charge, both of which are shown in Figure 9 for comparison. Overall, our Hall-MHD model prediction, especially the perpedicular velocity component that is governed by the ExB drift, agrees better with the derived PLS velocity data assuming heavy ions. This result is consistent with the finding obtained in the previous MHD modeling of Jia et al. (2009), both in support of the suggestion first made by Vasyliūnas and Eviatar (2000) that the plasma population detected by the Galileo PLS inside Ganymedes polar cap during the G2 flyby is composed mainly of heavy ions. 
Third, near the inbound magnetopause crossing, our model predicts higher plasma velocities than seen in both Frank et al. (1997b) and Collinson et al. (2018) results. These high speed flows in our model are associated with reconnection jets produced by magnetopause reconnection near the flanks, which appears to be intermittent. Therefore, the discrepancy between the model and data may be due in part to lack of high temporal resolution measurements, but clearly further studies and new observations are needed to confirm our model prediction.

\subsection{Upstream Reconnection}

Generally, sources of magnetic field variations during the magnetopause crossing include the Hall effect, FTEs, waves and instabilities. It is hard to predict theoretically the contribution from each part in different regions of a nonlinear system. Similarly to previous work of G8 flyby simulation (Tóth et al., 2016), by extracting the magnetic field from the time-accurate runs along the Galileo flyby trajectories, we can examine reconnectionrelated features in the magnetic field especially near the magnetopause. From this point forward, we will only discuss results from time-accurate MHD-EPIC simulations of the G8 and G28 flybys. The magnetic field comparisons from the MHD-EPIC of the G8 and G28 flyby simulation are shown in Figure 7(a) and Figure 8(a). A 20-minute simulation is sufficient to cover the inbound and outbound crossings for both G8 and G28 flybys in a continuous manner. Since there is no time variation in the driving conditions and flux transfer events are generated spontaneously, we have the freedom to shift the start time of the simulation relative to observations. The data outside the simulation time interval are compared with outputs from the same snapshot of the starting and ending points. Figure 7(a) shows the magnetic field comparison for the time-accurate G8 flyby MHDEPIC simulation, and Figure 7(b) shows the corresponding 3D field line tracing along Galileo's trajectory in one snapshot inside the PIC region at the upstream magnetopause. The oscillations in the three magnetic field components, especially $B_{y}$ during the G8 inbound crossing, are clear signatures of a transient FTE formed at the upstream magnetopause, while the overall magnetospheric structure is similar to that in the quasi-steady state. During the G8 flyby, Galileo was moving in the $+y$ direction (right to left in the Figure) from anti-Jovian side to sub-Jovian side. We identify flux ropes during the magnetopause inbound crossing with enhanced ion pressure at the center, and show field lines that just get reconnected from X-lines at the outbound crossing. In the observed magnetic field, there are spikes during the outbound crossing near $16: 03 \mathrm{UT}$, which is potentially a transient signature associated with a flux rope that is not entirely captured by the model at the same physical time. By going through all the snapshots in the 20min simulation, we do find flux ropes forming near the outbound magnetopause locations. In a complicated nonlinear system where no true steady state exists, it is not possible to reproduce exactly the same observation given the available observational constraints and the nature of flux rope formation. However, the fact that the overall trend and fluctuation as shown by time-accurate magnetic field comparisons and the power spectra (Figure 14 in our previous paper (Tóth et al., 2016) agree well with observations suggests that the MHD-EPIC model provides a very good description of the magnetic field structure at Ganymede.

Figure 8 displays the scenario for the G28 flyby in the same format as in Figure 7. In this case with near unity guide field $\left(B_{z} \sim B_{y}\right)$, the magnetic field tends to be more steady, even though one can still argue that there are small flux rope signatures during the magnetopause crossing. Actually, we do find flux ropes on the upstream magnetopause as shown in Figure 8(b); however, these appear mostly in regions away from the G28 flyby trajectory, so we cannot easily see them in the 1D synthetic magnetometer data.

Our model provides many more quantities beyond ideal MHD inside the PIC region. We plot magnetic field, pressure and velocity from PIC outputs on the $y=0$ merid- 
ional cut plane in Figure 10 at $t=420 \mathrm{~s}$. A pattern of quadrupolar Hall magnetic field centered at $(x, z)=\left(-1.9 R_{G}, 0.05 R_{G}\right)$ is clearly shown in Figure 10 (a), with a small electron diffusion region (Figure 10(e)) and a relatively large ion diffusion region (Figure 10(f)). There is also a fast electron drift in the out-of-plane (approximately $y$ ) direction across the magnetopause (Figure 10(d)), peaked at the reconnection sites due to curvature of $\mathbf{B}$ and the Hall effect. The deflection of electrons and ions in opposite directions at the magnetopause causes a Chapman-Ferraro like current pointing from Jovian side to anti-Jovian side $(+y$ to $-y)$. Corresponding in-plane electric field $E_{x}$ is shown in the left panel of Figure 11. The ambipolar structure of $E_{x}$ arises from the electronion charge separation at the magnetopause, which is both included in the Hall MHD and PIC model. Ions are being accelerated by this in-plane electric field, entering the reconnection exhaust across the separatrices.

\subsection{Ion Velocity Distribution}

With an embedded PIC model, we can obtain detailed information about the behavior of the electrons and ions directly by studying their velocity distributions near the $\mathrm{X}$-line. Due to the quasi-symmetry at the nose of the magnetopause, the generally used LMN boundary-normal coordinate system in reconnection studies is aligned with the Cartesian coordinates, where the normal direction $N$ is $-x$ direction, $L$ is $z$ direction, and outof-plane direction $M$ is $y$ direction, so it is convenient to plot model results in the simulation coordinates. Clear signatures of ion motion near the separatrices are nongyrotropic distributions (e.g. DeCoster and Frank (1979); Ashour-Abdalla et al. (1993); Frank L. A. and Kivelson (1994) for Earth tail reconnection, Burch and Phan (2016) for Earth dayside magnetopause reconnection). Based on the $y=0$ plane Hall electric field $E_{x}$ contours on the left of Figure 11, we select four box regions of the same size to extract the ion distribution functions in the inflow and outflow regions near the X-line in the G8 flyby simulation, where $E_{x} \sim E_{N}$, and $B_{z} \sim B_{L}$. In the Cartesian coordinates, approximately, $u_{x}$ is the inflow velocity, $u_{z}$ is the outflow velocity, and $u_{y}$ is the out-of-plane velocity. The ion gyroradius $r_{i}$ in the simulation has a maximum value of about $0.2 R_{G}$ with an average of $0.06 R_{G}$, thus the selected box region has a width of $\sim 0.5 r_{i}$ in the $x$ direction, and $\sim 1.8 r_{i}$ in the $z$ direction. The right-hand side panels of Figure 11 show the ion distributions in velocity phase space for the inflow and outflow regions, respectively, where the histograms are normalized by probability distribution separately. In the $u_{i x}-u_{i z}$ plots on the upper right, there are single peaks in the two inflow regions which represent the isotropic inflow streams, and double peaks in the outflow regions that represent the counterstreaming behavior from ion entry with anisotropy in parallel and perpendicular directions. Such counterstreaming behavior has also been observed in earlier local PIC and hybrid simulations of Earth-like reconnection (Drake et al., 2009; Hoshino et al., 1998; Arzner \& Scholer, 2001), and by Cluster spacecraft 1 in the Earth's magnetosheath (Phan et al., 2007). The $u_{i y}-u_{i z}$ plots are shown on bottom right. Moving from upstream into the magnetosphere across the X line (Region 1 to Region 2), we see that the two beams in the upstream merge and a crescent-shaped distribution forms on the magnetospheric side, which corresponds to the ion meandering motion in the diffusion region near the reconnection site (Lapenta et al., 2017; Bessho et al., 2016; Egedal et al., 2016). In the two outflow regions, there is a similar butterfly shape distribution in this $u_{i y}-u_{i x}$ cut, which represents a drift in the $y$ direction and anisotropy in temperature between the two perpendicular directions. The non-gyrotropic anisotropic distribution functions show that the embedded kinetic model can simulate the magnetic reconnection from first principle.

In the case of the G28 flyby simulation, we also check the ion distribution near the reconnection site (not shown here). There is evidence of ion crescent distribution in the perpendicular plane at the magnetospheric side of inflow region, even in a strong guide field case. 


\subsection{Energy Flux Density and Auroral Emission}

The particle information obtained from the PIC model allows us to calculate the energy flux densities in a self-consistent manner. Observations by the Hubble Space Telescope (HST) indicate that Ganymede possesses auroral emissions (Feldman et al., 2000). It is shown that the auroral oval lies very close to the polar cap boundary that separates the open and closed magnetic field lines (McGrath et al., 2013). On the upstream side, the open-closed field line boundary maps to the magnetopause where reconnection is taking place. It is, therefore, reasonable to expect that reconnection-produced energetic particles may contribute to the generation of aurora. Here we first calculate the energy flux densities using the particle information output from the PIC model to assess the contribution of magnetopause reconnection to the aurora emissions. In our simulation, the inner edge of the PIC box is set at $x=-1.125 R_{G}$. Although the PIC region set at the upstream magnetopause does not include the moon's surface, we can calculate the energy flux densities inside the PIC region and map them to the surface based on the magnetic field topology resolved by the global model under the assumption of flux conservation. Specifically, the procedure goes as follows:

1. Read particle information from PIC and magnetic field information from MHD.

2. For each particle in the selected region, interpolate the magnetic field at the particle locations and calculate the pitch angles.

3. Find the connectivities of magnetic field lines between particle locations and the moon's surface, and then compute the critical angles for the loss cone.

4. Select particles inside the loss cone, and calculate the energy flux densities along the field lines inside the PIC region.

5. Map the fluxes onto the surface along the field lines.

The most important assumptions in this analysis are the conservation of energy and magnetic flux. With the current model and the linear interpolation and integration approaches, the error in magnetic flux conservation is less than $5 \%$, which is sufficient for obtaining an overall distribution of flux densities and comparison with remote observations. The energetic particles inside the loss cone is about $4 \%$ of the total number of macroparticles in the sliced PIC region, with an average critical angle of roughly $20^{\circ}$. Besides, because of the size of PIC box, we are restricted to a finite portion of the surface area which has energetic particle precipitation from the PIC region.

The mapped energy flux densities for both ions and electrons at $t=280 \mathrm{~s}$ are shown in Figure 12 viewing from upstream. We can clearly see curves of peak flux density on the order of $10^{-7} \mathrm{~W} / \mathrm{cm}^{2}$ for both species in the northern and southern hemisphere. This is on the same order but slightly higher than a rough average polar cap precipitating energetic flux estimation given by Frank et al. (1997a) for the low-energy electron measurements between 0.5 and $3.0 \mathrm{keV}$ for G2 flyby, but about one order higher than the G7 flyby estimation assuming an invariant latitude of $\sim 45^{\circ}$ by Paranicas et al. (1999), probably due to the intensities of low-energy electrons over the polar cap. Both of the previous estimates use raw data from PLS measurements, with extrapolation fit over the energy range. Given the existence of aurora near the cusp region due to the precipitating electrons, concentrated energy fluxes with higher intensities than the surroundings are expected in the cusp. In both hemispheres, the largest flux density lies within approximately $\left[45^{\circ}, 55^{\circ}\right]$ latitude, which is consistent with Hubble observations of atomic oxygen emission by McGrath et al. (2013). For the $60^{\circ}$ in longitude on the trailing side that is covered by the upstream PIC box, the location of peak energetic fluxes obtained from our model correlates well with the location of the brightest emissions observed by the HST (Figure 3 in McGrath et al. (2013)). In our model, the energetic flux densities for electrons and ions inside the loss cone are on the same order of magnitude with an obvious asymmetry between Jovian/anti-Jovian $( \pm y)$ directions: generally larger for ions in the $-y$ direction and electron in the $+y$ direction. One plausible explanation for the 
asymmetry is the Hall effect: the separation of electron and ion motions causes a ChapmanFerraro like current across the magnetopause from left to right $(+y$ to $-y)$, thus the mapping of fluxes from the two species from the magnetosphere onto the surface follows the same pattern.

From Figure 4, we observe a high correlation of the open-closed field line boundary (shown in red) with the peak emission locations which connect to the "cusp" and upstream reconnection outflow regions. The bright peak line near the moon's surface can be caused by the magnetic mapping from the magnetosphere to the surface, or the distribution of energetic particles out of reconnection sites. To check which is the effective cause, we replaced the real energetic particle fluxes along the field lines with uniform flux and performed the same mapping. The result shows that even though there is still a single peak curve on the surface, the whole distribution has no sharp gradient like the ones shown in Figure 12. This experiment suggests that both the magnetic focusing and the distribution of energetic particles at the origins have influence on the distribution of the energetic flux densities near the surface.

For a brightness estimation, Payan et al. (2015) used a radially-averaged relation (Equation 5 in the paper) between the average electron number density, average collisional excitation rate and molecular oxygen column density. We apply the same idea in estimating the emission caused by reconnection process in the upstream, considering only the energetic electrons out of the upstream reconnection region. If we map these electrons with $\sim 1 \mathrm{keV}$ temperature inside the loss cone to the surface of Ganymede under $B / n$ conservation, we get a surface energetic electron number density $3 \mathrm{~cm}^{-3}$. The upper constraint on the polar region molecular oxygen column density is $N\left(O_{2}\right) \sim 4 \times$ $10^{14} \mathrm{~cm}^{-2}$ from Voyager observation (Broadfoot et al., 1981). The electron pitch angle distribution is expected to become roughly isotropic near the surface where the neutral oxygen density is high, so the length of the path of the electron is about $\sqrt{3} \approx 1.7$ times higher than the length of the field line. In addition, the field lines are inclined, which further increases the integrated column density along the trajectory of the electron, so we use $N^{\prime} \approx 8 \times 10^{14} \mathrm{~cm}^{-2}$ for the effective column density. With an average collisional excitation rate $\sim 5 \times 10^{-8} \mathrm{~cm}^{3} \mathrm{~s}^{-1}$ for $\mathrm{keV}$ electrons colliding with atomic oxygen at $1356 \AA$ emission wavelength (derived from (Payan et al., 2015)), these reconnection-accelerated electrons contribute to a maximum of $\sim 120 \mathrm{R}$ in the northern and southern hemisphere, which corresponds to $40 \%$ of the peak emission brightness observed by Feldman et al. (2000).

\section{Discussion}

Even though the magnetic field comparison is fairly good, our MHD-EPIC model clearly cannot reproduce every detailed feature observed by Galileo. Most of the discrepancies appear in the magnetopause crossings, and in general the simulation results look more smoother than observations. This may indicate that the second order schemes we applied to MHD and PIC are not fully capable of capturing the sharp changes at the feasible grid resolution. In the future, the nonsymmetric resistivity distribution, a putative ocean with high conductivity located between $150 \mathrm{~km}$ and $250 \mathrm{~km}$ depth (M. Kivelson et al., 2002; Saur et al., 2015) and new knowledge about the interior structure may intrigue the development of a more realistic resistivity model. Additionally, because of the intrinsic differences between Hall MHD and PIC model, a coupled PIC region produces different results from the Hall MHD model. As can be been in Figure 7(a) and Figure 8(a), the magnetic field comparisons are not as good as in the stand-alone Hall MHD simulations (Figure $6(\mathrm{e}, \mathrm{f})$ ).

Considering the reduction of the ion-electron mass ratio 100 in the PIC simulation, the electron results must be interpreted with care. Our grid resolution in the PIC domain is $0.2 d_{i} \sim 0.4 r_{i} \sim 2 d_{e}$, which is sufficient to resolve ion-scale physics but not enough 
to fully resolve electron-scale physics, especially near the electron diffusion region. Indeed, we do not find the crescent shape in the electron phase space distribution near the X-line reconnection sites. In the MHD-EPIC application to Earth's dayside magnetopause with an extra scaling factor included (Chen et al., 2017), the crescent distribution for electrons can be identified with a grid resolution of $0.5 d_{e}$ which is 4 times finer than our Ganymede's case. Based on our previous scaling studies (Tóth et al., 2017), we are confident that the global ion-scale dynamics is well captured by the MHD-EPIC model, while the modeling of electon-scale physics can be improved by further increasing the grid resolution.

Eviatar et al. (2001) investigated the cause of Ganymede's auroral emission and discussed the possible mechanisms for local acceleration required to heat the electrons. In the analog to the terrestrial case, they stressed that the orders of magnitude smaller atmosphere column density at Ganymede than that at Earth leads to the penetration of energetic electrons almost totally without collisions. Thus the secondary electrons shall not play an important role in Ganymede's aurora emission, and we do not include them in the estimation. Our calculation from PIC simulation on the upstream side shows that hot electrons coming from the reconnection site can contribute a certain fraction of the peak emission brightness, which is consistent with the estimation of continuous aurora by Eviatar et al. (2001), and tends to favor the suggested local acceleration mechanisms proposed thereafter. A recent Hubble observation analysis by Molyneux et al. (2018) suggests an optically thick $\mathrm{O}$ atmosphere besides $\mathrm{O}_{2}$, with a ratio of $\mathrm{O} / \mathrm{O}_{2} \sim 10 \%$. Since $\mathrm{O}$ has a smaller electron impact cross section than that of $\mathrm{O}_{2}$, the estimated direct reconnectioncontributed emission could only be smaller. Besides, upstream reconnection as shown by our model also produces ion energetic fluxes with the same order of magnitude as electron energetic fluxes. However the ion contribution is negligible due to smaller thermal velocities.

From plasma observations (M. G. Kivelson et al., 2004), we know there are at least two ion populations in the ambient Jovian environment: thermal and hot components. For a single fluid model like we have shown in this paper, we have to make assumptions in prescribing a single upstream ion temperature that results in a total particle pressure consistent with the observations. A more realistic simulation left for future work should be separating the ion populations using a multi-fluid MHD model as well as introducing more particle species inside the PIC region. As a natural extension to the current MHD-EPIC coupling at the upstream magnetopause, we can extend the PIC region to cover the majority of Ganymede's magnetosphere such that we can have a global-scale kinetic model. Future work may also include a even more consistent coupling between multi-fluid MHD, or even higher moments MHD, and the PIC model.

\section{Conclusions}

In this study, we have presented and improved MHD-EPIC model of Ganymede's magnetosphere. With an adaptive stretched spherical MHD grid, an incorporated magnetic diffusion solver down to the core region, and a new energy-conserving PIC scheme, the simulations show a dynamic magnetosphere with reconnection-related features. Key findings from the current simulations are:

1. Single fluid MHD-EPIC model is able to capture the global structure of magnetic field in Ganymede's sub-Alfvénic magnetosphere, shown by the quasi-steady comparison over all six Galileo flybys. Intermittent FTEs are generated even under steady upstream conditions, signatures of which can be potentially captured by in-situ magnetometer observations.

2. Ion-scale physics including nongyrotropic and non-isotropic effects are fully resolved in the PIC domain covering the majority of the upstream magnetopause. Crescent- 
shape ion distribution is found on the magnetospheric side of the upstream reconnection.

3. The energetic flux densities from upstream reconnection obtained directly from the PIC simulation are about $10^{-7} \mathrm{~W} / \mathrm{cm}^{2}$. A quantitative estimation on the auroral emission shows that upstream reconnection can contribute for up to about half of the peak brightness.

With the upcoming JUICE mission, the embedded kinetic models will be more useful in providing insights into Ganymede's dynamic system.

\section{Acknowledgments}

The authors thank Michael Liemohn for the useful discussions on the auroral emission estimation. The authors would also like to thank the reviewers for their useful comments. This research is supported by INSPIRE and NASA Solar System Workings program through grant NNX15AH28G. Computational resources supporting this work were provided on the Blue Waters super computer by the NSF PRAC grant ACI-1640510.

The SWMF code (including BATS-R-US and iPIC3D) is publicly available through the csem.engin.umich.edu/tools/swmf web site after registration. The simulation data presented in the current research is available on doi:10.7302/q87q-k740.

\section{References}

Anderson, J., Lau, E., Sjogren, W., Schubert, G., \& Moore, W. (1996). Gravitational constraints on the internal structure of ganymede. Nature, 384(6609), 541.

Arzner, K., \& Scholer, M. (2001). Kinetic structure of the post plasmoid plasma sheet during magnetotail reconnection. Journal of Geophysical Research: Space Physics, 106(A3), 3827-3844.

Ashour-Abdalla, M., Berchem, J. P., Büchner, J., \& Zelenyi, L. M. (1993). Shaping of the magnetotail from the mantle: Global and local structuring. Journal of Geophysical Research: Space Physics, 98(A4), 5651-5676.

Bessho, N., Chen, L.-J., \& Hesse, M. (2016). Electron distribution functions in the diffusion region of asymmetric magnetic reconnection. Geophysical Research Letters, 43(5), 1828-1836.

Broadfoot, A., Sandel, B., Shemansky, D., McConnell, J., Smith, G., Holberg, J., ... Bertaux, J. (1981). Overview of the voyager ultraviolet spectrometry results through jupiter encounter. Journal of Geophysical Research: Space Physics, 86 (A10), 8259-8284.

Burch, J., \& Phan, T. (2016). Magnetic reconnection at the dayside magnetopause: Advances with mms. Geophysical Research Letters, 43(16), 8327-8338.

Burch, J., Torbert, R., Phan, T., Chen, L.-J., Moore, T., Ergun, R., ... others (2016). Electron-scale measurements of magnetic reconnection in space. Science, 352(6290), aaf2939.

Chen, Y., \& Toth, G. (2018). Gauss's law satisfying energy-conserving semi-implicit particle-in-cell method. arXiv preprint arXiv:1808.05745.

Chen, Y., Tóth, G., Cassak, P., Jia, X., Gombosi, T. I., Slavin, J. A., ... Henderson, M. G. (2017). Global three-dimensional simulation of earth's dayside reconnection using a two-way coupled magnetohydrodynamics with embedded particle-in-cell model: Initial results. Journal of Geophysical Research: Space Physics, 122(10), 10-318.

Collinson, G., Paterson, W. R., Bard, C., Dorelli, J., Glocer, A., Sarantos, M., \& Wilson, R. (2018). New results from galileo's first flyby of ganymede: Reconnection-driven flows at the low-latitude magnetopause boundary, cross- 
ing the cusp, and icy ionospheric escape. Geophysical Research Letters, 45(8), $3382-3392$.

Daldorff, L. K., Tóth, G., Gombosi, T. I., Lapenta, G., Amaya, J., Markidis, S., \& Brackbill, J. U. (2014). Two-way coupling of a global hall magnetohydrodynamics model with a local implicit particle-in-cell model. Journal of Computational Physics, 268, 236-254.

DeCoster, R. J., \& Frank, L. A. (1979). Observations pertaining to the dynamics of the plasma sheet. Journal of Geophysical Research: Space Physics, 84(A9), 5099-5121.

Dedner, A., Kemm, F., Kröner, D., Munz, C.-D., Schnitzer, T., \& Wesenberg, M. (2002). Hyperbolic divergence cleaning for the mhd equations. Journal of Computational Physics, 175(2), 645-673.

Dorelli, J. C., Glocer, A., Collinson, G., \& Tóth, G. (2015). The role of the hall effect in the global structure and dynamics of planetary magnetospheres: Ganymede as a case study. Journal of Geophysical Research: Space Physics, 120(7), 5377-5392.

Drake, J., Swisdak, M., Phan, T., Cassak, P., Shay, M., Lepri, S., ... Zurbuchen, T. (2009). Ion heating resulting from pickup in magnetic reconnection exhausts. Journal of Geophysical Research: Space Physics, 114(A5).

Duling, S., Saur, J., \& Wicht, J. (2014). Consistent boundary conditions at nonconducting surfaces of planetary bodies: Applications in a new ganymede mhd model. Journal of Geophysical Research: Space Physics, 119(6), 4412-4440.

Eastwood, J., Phan, T., Cassak, P., Gershman, D., Haggerty, C., Malakit, K., .. others (2016). Ion-scale secondary flux ropes generated by magnetopause reconnection as resolved by mms. Geophysical research letters, 43(10), 47164724.

Egedal, J., Le, A., Daughton, W., Wetherton, B., Cassak, P., Chen, L.-J., ... others (2016). Spacecraft observations and analytic theory of crescent-shaped electron distributions in asymmetric magnetic reconnection. Physical review letters, $117(18), 185101$.

Eviatar, A., Strobel, D. F., Wolven, B. C., Feldman, P. D., McGrath, M. A., \& Williams, D. J. (2001). Excitation of the ganymede ultraviolet aurora. The Astrophysical Journal, 555(2), 1013.

Fatemi, S., Poppe, A., Khurana, K., Holmström, M., \& Delory, G. (2016). On the formation of ganymede's surface brightness asymmetries: Kinetic simulations of ganymede's magnetosphere. Geophysical Research Letters, 43(10), 47454754 .

Feldman, P. D., McGrath, M. A., Strobel, D. F., Moos, H. W., Retherford, K. D., \& Wolven, B. C. (2000). Hst/stis ultraviolet imaging of polar aurora on ganymede. The Astrophysical Journal, 535(2), 1085.

Frank, L., Paterson, W., Ackerson, K., \& Bolton, S. (1997a). Low-energy electron measurements at ganymede with the galileo spacecraft: Probes of the magnetic topology. Geophysical research letters, 24(17), 2159-2162.

Frank, L., Paterson, W., Ackerson, K., \& Bolton, S. (1997b). Outflow of hydrogen ions from ganymede. Geophysical research letters, 24(17), 2151-2154.

Frank L. A., W. R. P., \& Kivelson, M. G. (1994). Observations of nonadiabatic acceleration of ions in earth's magnetotail. Journal of Geophysical Research: Space Physics.

Hoshino, M., Mukai, T., Yamamoto, T., \& Kokubun, S. (1998). Ion dynamics in magnetic reconnection: Comparison between numerical simulation and geotail observations. Journal of Geophysical Research: Space Physics, 103(A3), 4509-4530.

Jia, X., Slavin, J. A., Gombosi, T. I., Daldorff, L. K., Toth, G., \& Holst, B. (2015). Global mhd simulations of mercury's magnetosphere with coupled planetary interior: Induction effect of the planetary conducting core on the global inter- 
action. Journal of Geophysical Research: Space Physics, 120(6), 4763-4775.

Jia, X., Walker, R. J., Kivelson, M. G., Khurana, K. K., \& Linker, J. A. $\quad$ (2008). Three-dimensional mhd simulations of ganymede's magnetosphere. Journal of Geophysical Research: Space Physics, 113(A6).

Jia, X., Walker, R. J., Kivelson, M. G., Khurana, K. K., \& Linker, J. A. Properties of ganymede's magnetosphere inferred from improved threedimensional mhd simulations. Journal of Geophysical Research: Space Physics, $114(\mathrm{~A} 9)$.

Jia, X., Walker, R. J., Kivelson, M. G., Khurana, K. K., \& Linker, J. A. $\quad$ (2010). Dynamics of ganymede's magnetopause: Intermittent reconnection under steady external conditions. Journal of Geophysical Research: Space Physics, 115(A12).

Kivelson, M., Khurana, K., Russell, C., Walker, R., et al. (1996). Discovery of ganymede's magnetic field by the galileo spacecraft. Nature, $384(6609), 537$.

Kivelson, M., Khurana, K., \& Volwerk, M. (2002). The permanent and inductive magnetic moments of ganymede. Icarus, 157(2), 507-522.

Kivelson, M. G., Bagenal, F., Kurth, W. S., Neubauer, F. M., Paranicas, C., \& Saur, J. (2004). Magnetospheric interactions with satellites. Jupiter: The planet, satellites and magnetosphere, 513-536.

Lapenta, G. (2017). Exactly energy conserving semi-implicit particle in cell formulation. Journal of Computational Physics, 334, 349-366.

Lapenta, G., Berchem, J., Zhou, M., Walker, R., El-Alaoui, M., Goldstein, M., ... others (2017). On the origin of the crescent-shaped distributions observed by mms at the magnetopause. Journal of Geophysical Research: Space Physics, 122(2), 2024-2039.

Ma, Y., Russell, C. T., Toth, G., Chen, Y., Nagy, A. F., Harada, Y., ... others (2018). Reconnection in the martian magnetotail: Hall-mhd with embedded particle-in-cell simulations. Journal of Geophysical Research: Space Physics, 123(5), 3742-3763.

Markidis, S., Lapenta, G., et al. (2010). Multi-scale simulations of plasma with ipic3d. Mathematics and Computers in Simulation, 80(7), 1509-1519.

McGrath, M. A., Jia, X., Retherford, K., Feldman, P. D., Strobel, D. F., \& Saur, J. (2013). Aurora on ganymede. Journal of Geophysical Research: Space Physics, $118(5), 2043-2054$.

Molyneux, P., Nichols, J., Bannister, N., Bunce, E., Clarke, J., Cowley, S., .. Paty, C. (2018). Hubble space telescope observations of variations in ganymede's oxygen atmosphere and aurora. Journal of Geophysical Research: Space Physics, 123(5), 3777-3793.

Neubauer, F. M. (1998). The sub-alfvénic interaction of the galilean satellites with the jovian magnetosphere. Journal of Geophysical Research: Planets, 103(E9), 19843-19866.

Paranicas, C., Paterson, W., Cheng, A., Mauk, B., McEntire, R., Frank, L., \& Williams, D. (1999). Energetic particle observations near ganymede. Journal of Geophysical Research: Space Physics, 104(A8), 17459-17469.

Paty, C., Paterson, W., \& Winglee, R. (2008). Ion energization in ganymedes magnetosphere: Using multifluid simulations to interpret ion energy spectrograms. Journal of Geophysical Research: Space Physics, 113(A6).

Paty, C., \& Winglee, R. (2004). Multi-fluid simulations of ganymede's magnetosphere. Geophysical research letters, $31(24)$.

Paty, C., \& Winglee, R. (2006). The role of ion cyclotron motion at ganymede: Magnetic field morphology and magnetospheric dynamics. Geophysical research letters, 33(10).

Payan, A., Paty, C., \& Retherford, K. (2015). Uncovering local magnetospheric processes governing the morphology and variability of ganymede's aurora using three-dimensional multifluid simulations of ganymede's magnetosphere. 
Journal of Geophysical Research: Space Physics, 120(1), 401-413.

Phan, T., Paschmann, G., Twitty, C., Mozer, F., Gosling, J., Eastwood, J., ... Lucek, E. (2007). Evidence for magnetic reconnection initiated in the magnetosheath. Geophysical Research Letters, 34 (14).

Powell, K. G., Roe, P. L., Linde, T. J., Gombosi, T. I., \& De Zeeuw, D. L. (1999). A solution-adaptive upwind scheme for ideal magnetohydrodynamics. Journal of Computational Physics, 154(2), 284-309.

Russell, C. T., \& Elphic, R. (1978). Initial isee magnetometer results: Magnetopause observations. Space Science Reviews, 22(6), 681-715.

Saur, J., Duling, S., Roth, L., Jia, X., Strobel, D. F., Feldman, P. D., ... others (2015). The search for a subsurface ocean in ganymede with hubble space telescope observations of its auroral ovals. Journal of Geophysical Research: Space Physics, 120(3), 1715-1737.

Schubert, G., Zhang, K., Kivelson, M. G., \& Anderson, J. D. (1996). The magnetic field and internal structure of ganymede. Nature, 384(6609), 544.

Sokolov, I., Timofeev, E., Sakai, J., \& Takayama, K. $\quad$ (1999, Dec 01). $\quad$ On shock-capturing schemes using artificial wind. Shock Waves, 9(6), 423427. Retrieved from https://doi.org/10.1007/s001930050173 doi: $10.1007 / \mathrm{s} 001930050173$

Southwood, D., Farrugia, C., \& Saunders, M. (1988). What are flux transfer events? Planetary and space science, 36(5), 503-508.

Tóth, G., Chen, Y., Gombosi, T. I., Cassak, P., Markidis, S., \& Peng, I. B. (2017). Scaling the ion inertial length and its implications for modeling reconnection in global simulations. Journal of Geophysical Research: Space Physics, 122(10).

Tóth, G., Jia, X., Markidis, S., Peng, I. B., Chen, Y., Daldorff, L. K., ... others (2016). Extended magnetohydrodynamics with embedded particle-in-cell simulation of ganymede's magnetosphere. Journal of Geophysical Research: Space Physics, 121(2), 1273-1293.

Tóth, G., Ma, Y., \& Gombosi, T. I. (2008). Hall magnetohydrodynamics on blockadaptive grids. Journal of Computational Physics, 227(14), 6967-6984.

Tóth, G., Van der Holst, B., Sokolov, I. V., De Zeeuw, D. L., Gombosi, T. I., Fang, F., ... others (2012). Adaptive numerical algorithms in space weather modeling. Journal of Computational Physics, 231 (3), 870-903.

Vasyliūnas, V. M., \& Eviatar, A. (2000). Outflow of ions from ganymede: A reinterpretation. Geophysical research letters, 27(9), 1347-1349. 
Table 1. Simulation Parameters for Galileo's Six Close Encounters

\begin{tabular}{|c|c|c|c|c|c|c|c|c|c|c|c|}
\hline \multirow[b]{2}{*}{ Flyby } & \multicolumn{3}{|c|}{ Ganymede's Dipole Moment } & \multirow[b]{2}{*}{$B_{x}^{b k}$} & \multirow[b]{2}{*}{$B_{y}^{b k}$} & \multirow[b]{2}{*}{$B_{z}^{b k}$} & \multicolumn{5}{|c|}{ Background Flow } \\
\hline & $M_{z}[n T]$ & $M_{y}[n T]$ & $M_{x}[n T]$ & & & & $v[\mathrm{~km} / \mathrm{s}]$ & $\rho\left[\mathrm{amu} / \mathrm{cm}^{3}\right]$ & $P[n P a]$ & $M_{A}$ & $\beta$ \\
\hline G1 & -716.8 & 82.5 & -24.7 & 6 & -79 & -79 & 140 & 28 & 1.9 & 0.30 & 0.38 \\
\hline $\mathrm{G} 2$ & -716.8 & 80.0 & -29.3 & 17 & -73 & -85 & 140 & 28 & 1.9 & 0.30 & 0.38 \\
\hline G7 & -716.8 & 14.0 & -20.9 & -3 & 84 & -76 & 130 & 28 & 1.9 & 0.28 & 0.37 \\
\hline G8 & -716.8 & 51.8 & -18.0 & -10 & -6 & -86 & 140 & 56 & 3.8 & 0.55 & 1.60 \\
\hline G28 & -716.8 & 17.0 & -19.3 & -7 & 78 & -76 & 140 & 28 & 1.9 & 0.31 & 0.41 \\
\hline G29 & -716.8 & 84.2 & -18.4 & -9 & -83 & -79 & 140 & 28 & 1.9 & 0.30 & 0.36 \\
\hline
\end{tabular}

Table 2. Boundary condiitons

\begin{tabular}{cclcr}
\hline & Core Boundary $\left(r=0.5 R_{G}\right)$ & Surface Boundary $\left(r=1 R_{G}\right)$ & \multicolumn{2}{c}{ Outer Boundary (box) } \\
\cline { 3 - 4 } & & upstream, downstream & other \\
\hline$\rho$ & fixed, $550 \mathrm{amu} / \mathrm{cm}^{3}$ & fixed & float \\
$p$ & fixed, $0.115 \mathrm{nPa}$ & fixed & float \\
$p_{e}$ & fixed, $0.01 \mathrm{nPa}$ & fixed & float \\
$\mathbf{V}$ & & fixed & float \\
$\mathbf{B}$ & & fixed & float \\
\hline
\end{tabular}

Figure 1. On the top shows the whole simulation domain is a cube with edge length $200 R_{G}$ centered around Ganymede cut out of a stretched spherical grid. On the bottom is the grid structure near Ganymede shown by the $y=0$ and $z=0$ cuts. The inner boundary is represented by a sphere with radius $0.5 R_{G}$. The distance between neighboring purple balls is $1 R_{G}$. Adaptive mesh refinement is applied up to 2 levels near and within the magnetosphere. The color represents $B_{z}$ strengths in the two cut planes.

Figure 2. Sketch of the boundaries in the MHD model. 1 represents the core boundary at $r=$ $0.5 R_{G}, 2$ represents the surface boundary at $r=1 R_{G}$, and 3 represents the outer boundary. 
Figure 3. Radial profile of the spherical-symmetric resistivity used in the model. Values in each cell is interpolated linearly from specified points. The resistivity is set to 0 for region $r>1.05 R_{G}$.

figures/box_G8_view.png

Figure 4. (a) 3D front view of Ganymede's magnetopause from G8 flyby simulation. The dark blue sphere represents the moon's surface at $r=1 R_{G}$, the red surface represents the open-closed field line boundary, and the yellow surface displays the Alfvén wing that encloses Ganymede's magnetosphere. Field lines on the upstream passing through the $x=-1.95 R_{G}, z=0 R_{G}$ line are shown with black arrow lines. The PIC box at the upstream of magneopause is indicated with blue lines. (b) 3D anti-Jovian side view of Ganymede's magnetopause from G8 flyby simulation. Field lines are traced along $y=0 R_{G}, z=0 R_{G}$ line at the upstream and tail region, and also over the surface of the moon. The Galileo trajectory is plotted in green. 
figures/box_G28_view.png

Figure 5. a) 3D front view of Ganymede's magnetopause from G28 flyby simulation. Field lines on the upstream are traced along $x=-1.8 R_{G}, z=0 R_{G}$ line. (b) 3D anti-Jovian side view of Ganymede's magnetopause from G28 flyby simulation. Field lines are traced along $y=0 R_{G}, z=0 R_{G}$ line at the upstream and tail region, and also over the surface of the moon. The Galileo trajectory is plotted in green.

Figure 6. Magnetic field comparison with Galileo observation for quasi-steady state simulations of all six flybys. In each subplot, the Galileo magnetometer measurements are shown as black solid lines and the simulation results are plotted as red solid lines.

Figure 7. (a) Magnetic field extracted along the Galileo trajectory from 20 min time-accurate MHD-EPIC simulation of G8 flyby inside the PIC box region. The black line is the observations and the red line is the simulation results. Signatures of flux rope can be identified during the inbound crossing of magnetopause between $15: 51 \sim 15: 52$ UT, where sharp rotations of magnetic field, especially in the $B_{y}$ component, are present. (b) 3D topology of the magnetic field lines traced along the Galileo trajectory (shown by the white dots) inside the upstream PIC box at 15:51 UT. The colors of the field lines show the ion pressure. 
Figure 8. (a) Magnetic field extracted along the Galileo trajectory from 20 min time-accurate MHD-EPIC simulation of G28 flyby inside the PIC box region. The black line is the observations and the red line is the simulation results. (b) 3D topology of the magnetic field lines traced along the Galileo trajectory (shown by the white dots) inside the upstream PIC box. The colors of the field lines show the ion pressure.

Figure 9. Comparisons of parallel and perpendicular components of the plasma bulk flow velocities relative to the magnetic field for the G2 flyby. The PLS plasma data are obtained from (a) Frank et al. (1997b) and (b) Collinson et al. (2018), while the simulation data presented in red lines are from steady state Hall MHD. In each panel, the blue dots represent bulk flows derived from the PLS measurements for heavy ions with mass-per-charge $(M / Q)=16$. The gray dots are the calculated moments assuming $\mathrm{M} / \mathrm{Q}=1$, and the black dots represent bulk flows assuming $\mathrm{M} / \mathrm{Q}=16$. The two vertical dashed lines mark the inbound and the outbound magnetopause crossings identified from the magnetic field data, respectively.

Figure 10. Contour plots in the meridional plane $y=0$ containing the upstream magnetopause from time-accurate G8 simulation at $t=420 \mathrm{~s}$. The color contours represent (a) $B_{y}$ (Hall magnetic field); (b) $P_{e}$ (Electron pressure); (c) $P_{i}$ (Ion pressure); (d) $U_{e y}$ (Electron velocity in y-direction); (e) $U_{e z}$ (Electron velocity in z-direction); (f) $U_{i z}$ (Ion velocity in z-direction). All the velocities are normalized to the upstream Alfvén speed of $253 \mathrm{~km} / \mathrm{s}$.

Figure 11. Ion velocity distributions in normalized density near the upstream reconnection site from G8 flyby simulation at $t=420 \mathrm{~s}$. The colored contours on the left show the electric field $E_{x}$ overlaid with magnetic field lines in white, with four sample boxes (1 upstream inflow region, 2 magnetosphere inflow region, 3,4 outflow region) showing sampling locations used to extract the ion phase space distributions in $u_{i x}-u_{i z}$ (four upper right panels) and $u_{i y}-u_{i x}$ (four bottom right panels). The boxes extend in $y$ direction from $-0.08 R_{G}$ to $0.08 R_{G}$. All the velocities are normalized to the upstream Alfvén speed.

Figure 12. Energy flux densities mapped from PIC region onto Ganymede's surface for (a) ions and (b) electrons, respectively. This is a view from upstream, where $180^{\circ} \mathrm{E}$ points towards $-x$, and $90^{\circ} E$ points towards $+y$ (Jupiter) in GPhiO system. The mapping regions are limited to mid latitudes between approximately $150^{\circ} \mathrm{E}$ to $150^{\circ} \mathrm{W}$ due to the size of the PIC box. 

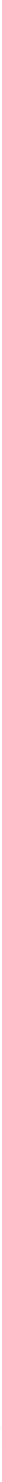
Figure 2.
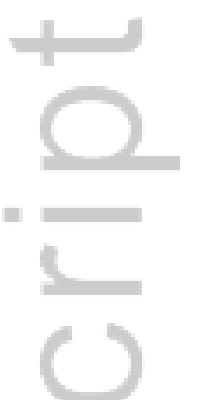

$\infty$
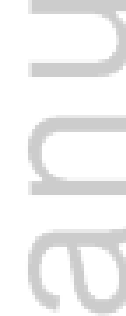

This article is protected by copyright. All rights reserved. 


\section{(3) $100 R_{G}$}

\section{$174 \mathrm{R}_{\mathrm{G}}$}

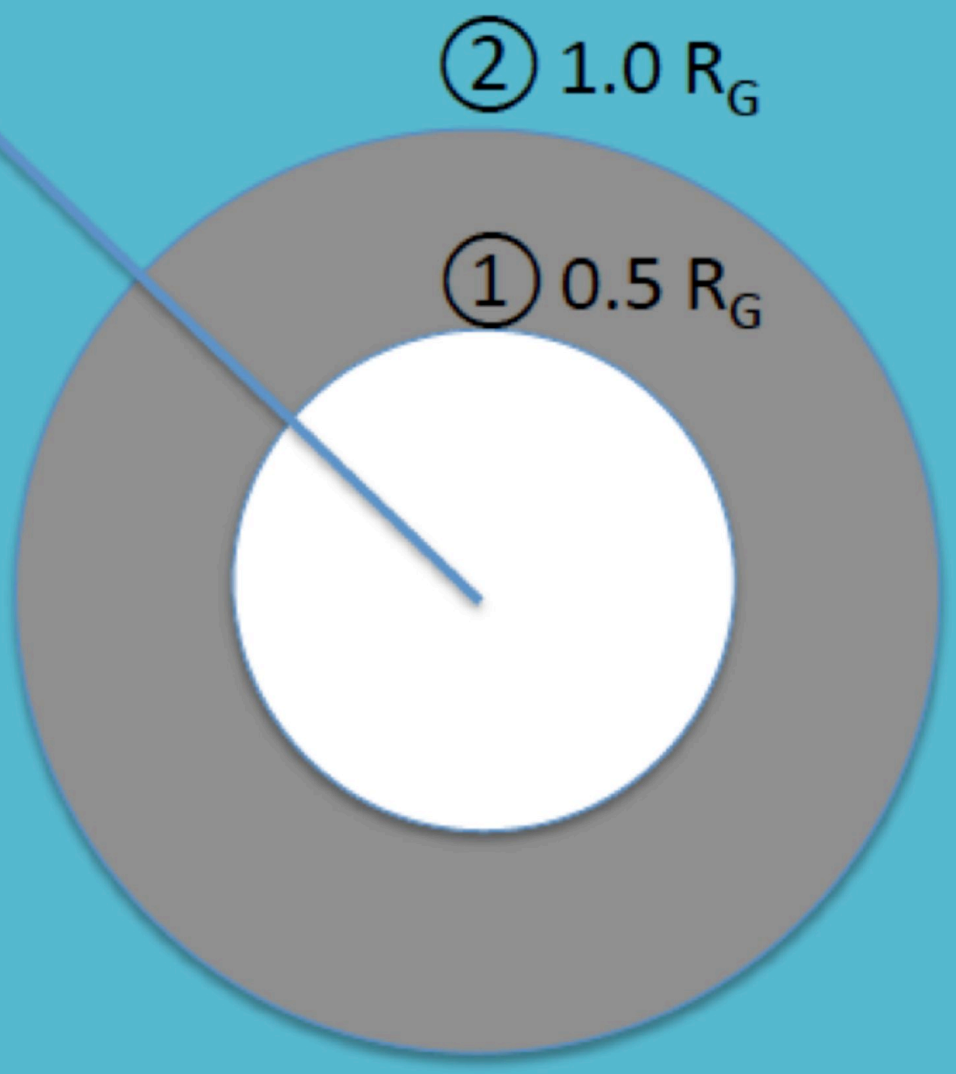

This article is protected by copyright. All rights reserved. 


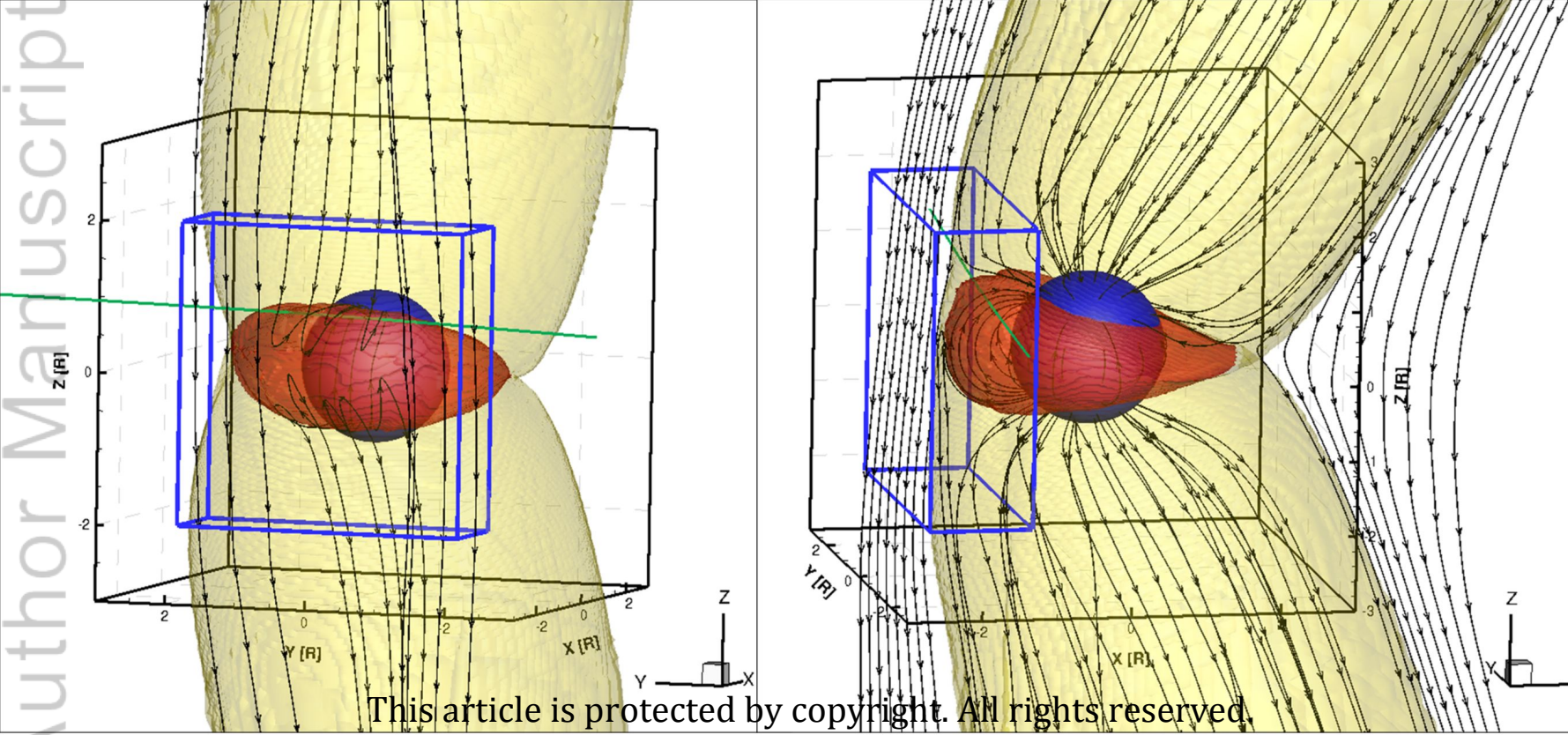


Figure 5.
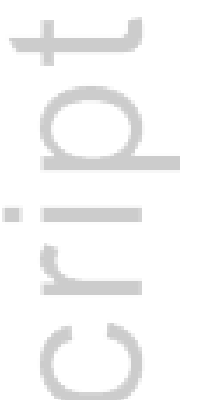

$\infty$
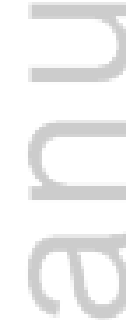

This article is protected by copyright. All rights reserved. 


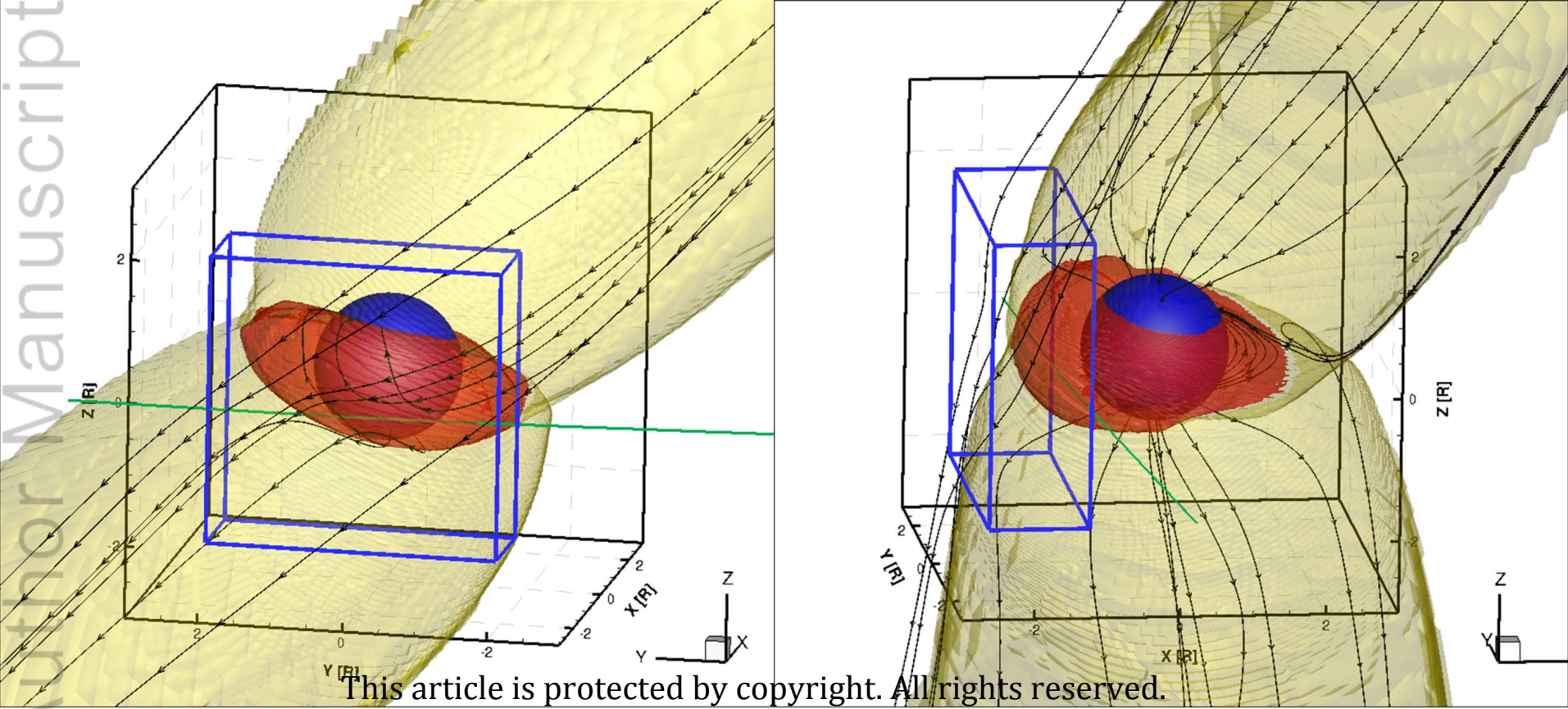

(a)

(b) 
(a)

Galileo G1 Flyby Magnetic field

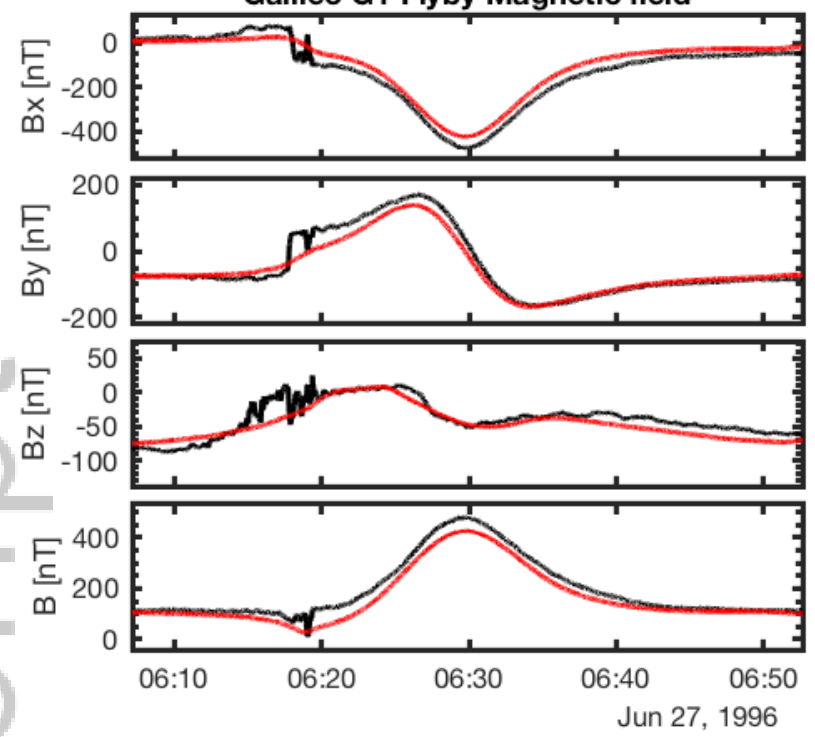

(c)

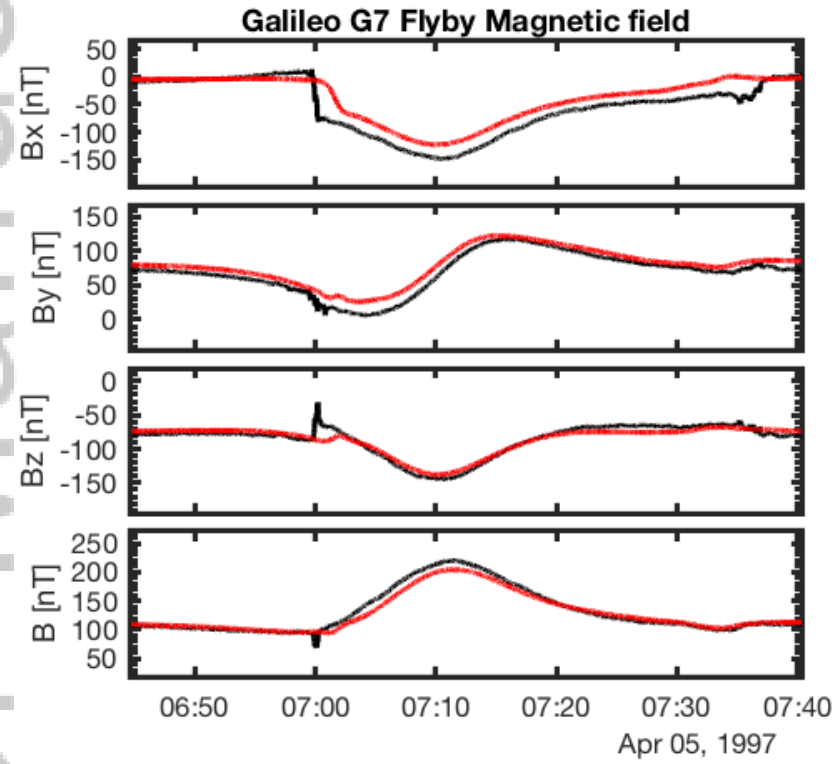

(e)

Galileo G28 Flyby Magnetic field

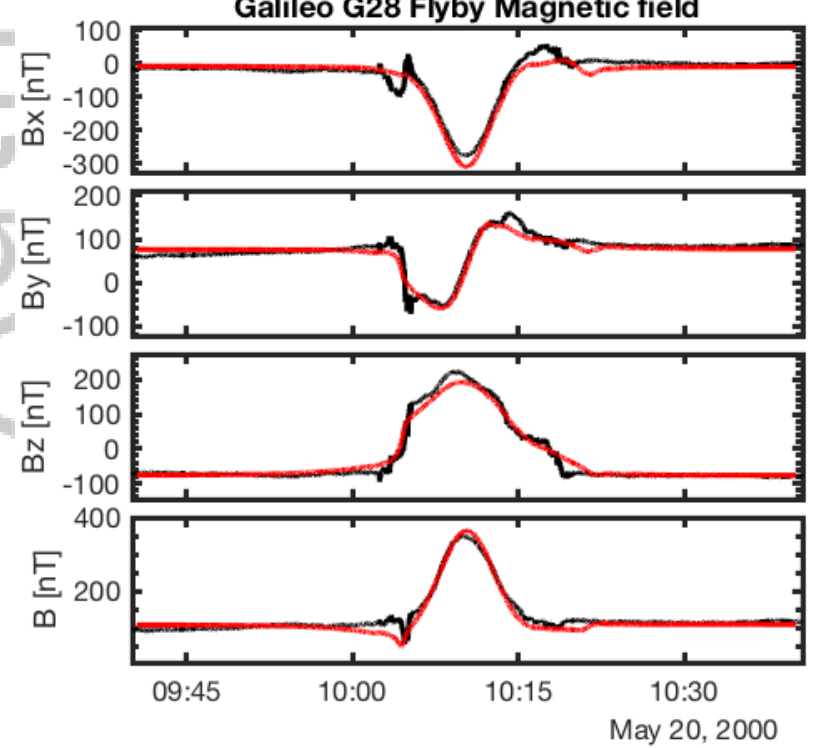

(b)

Galileo G2 Flyby Magnetic field

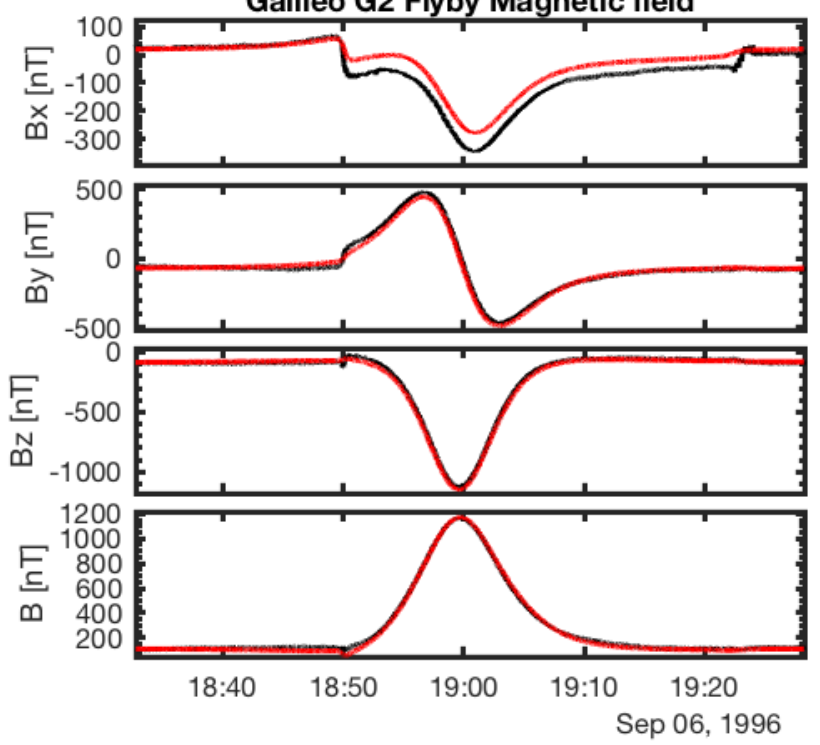

(d)

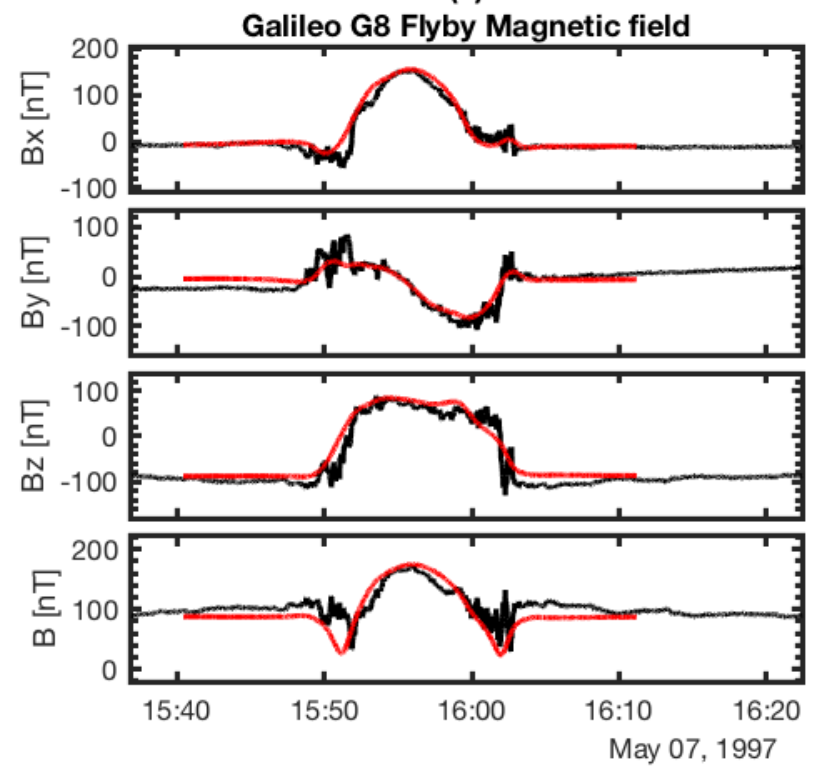

(f)

Galileo G29 Flyby Magnetic field
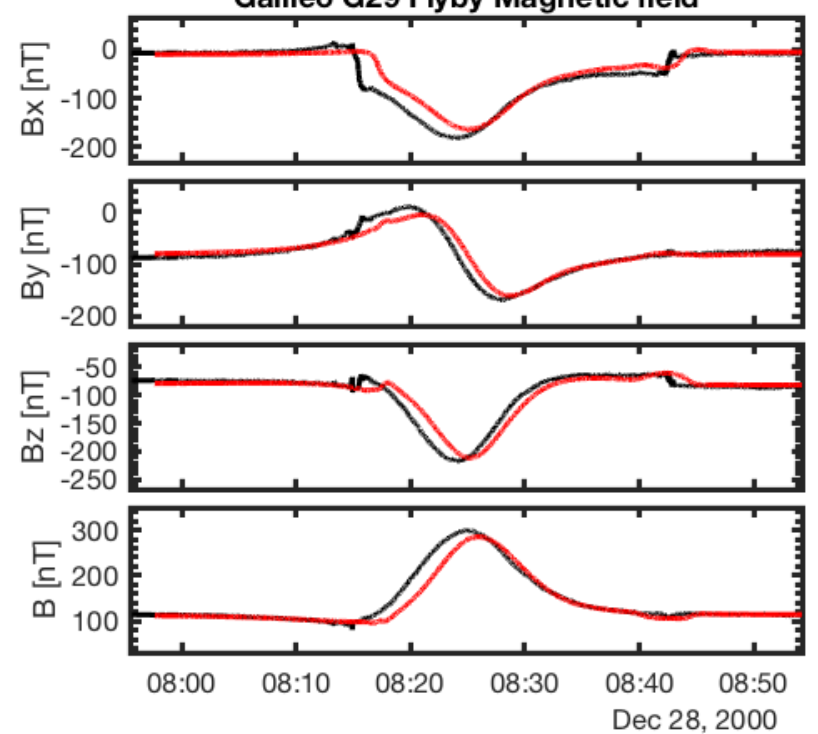

This article is protected by copyright. All rights reserved. 

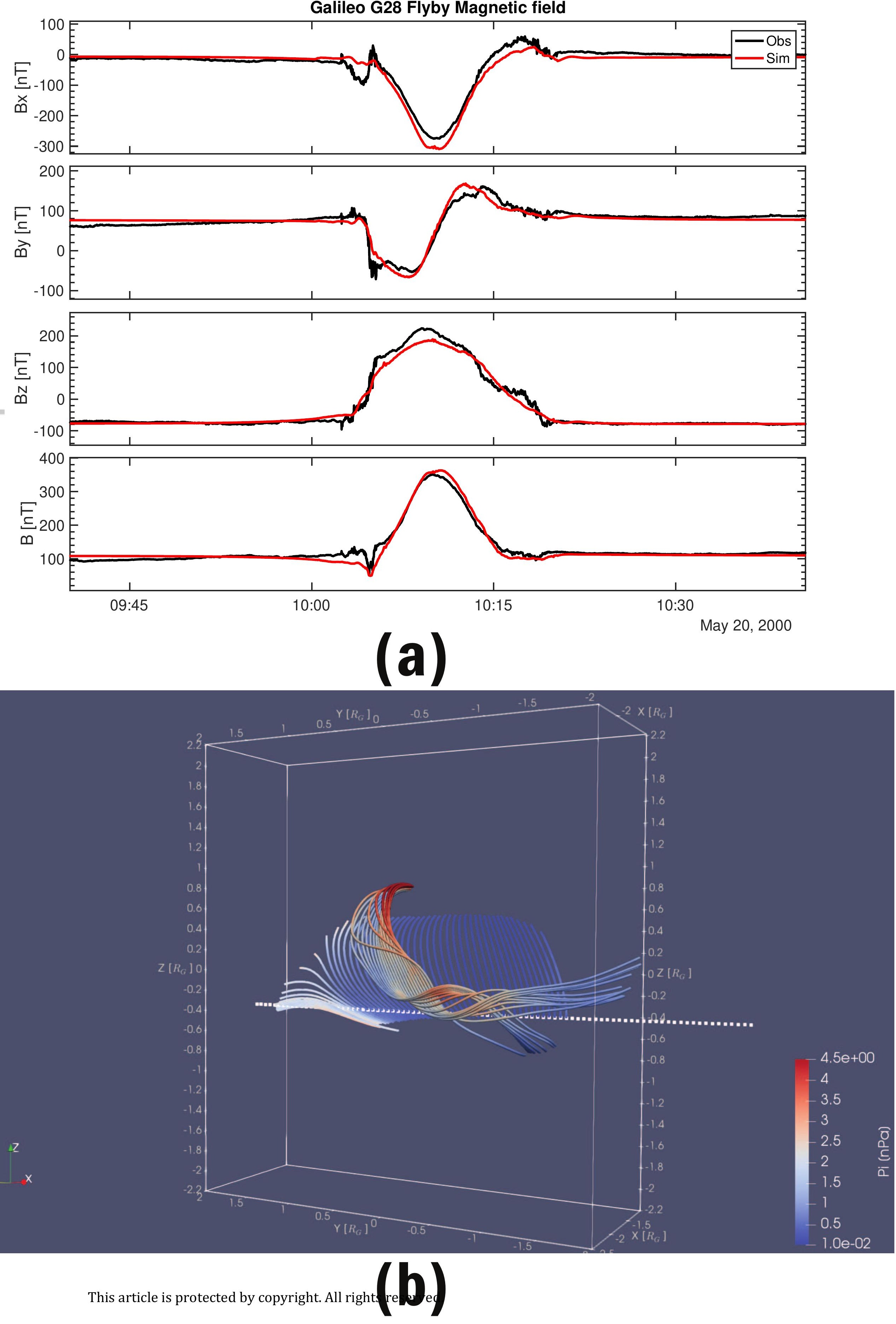
Figure 9.
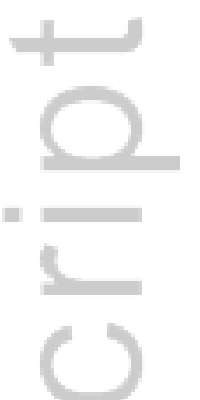

$\infty$
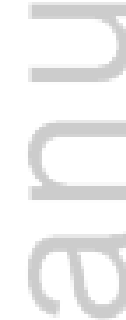

This article is protected by copyright. All rights reserved. 


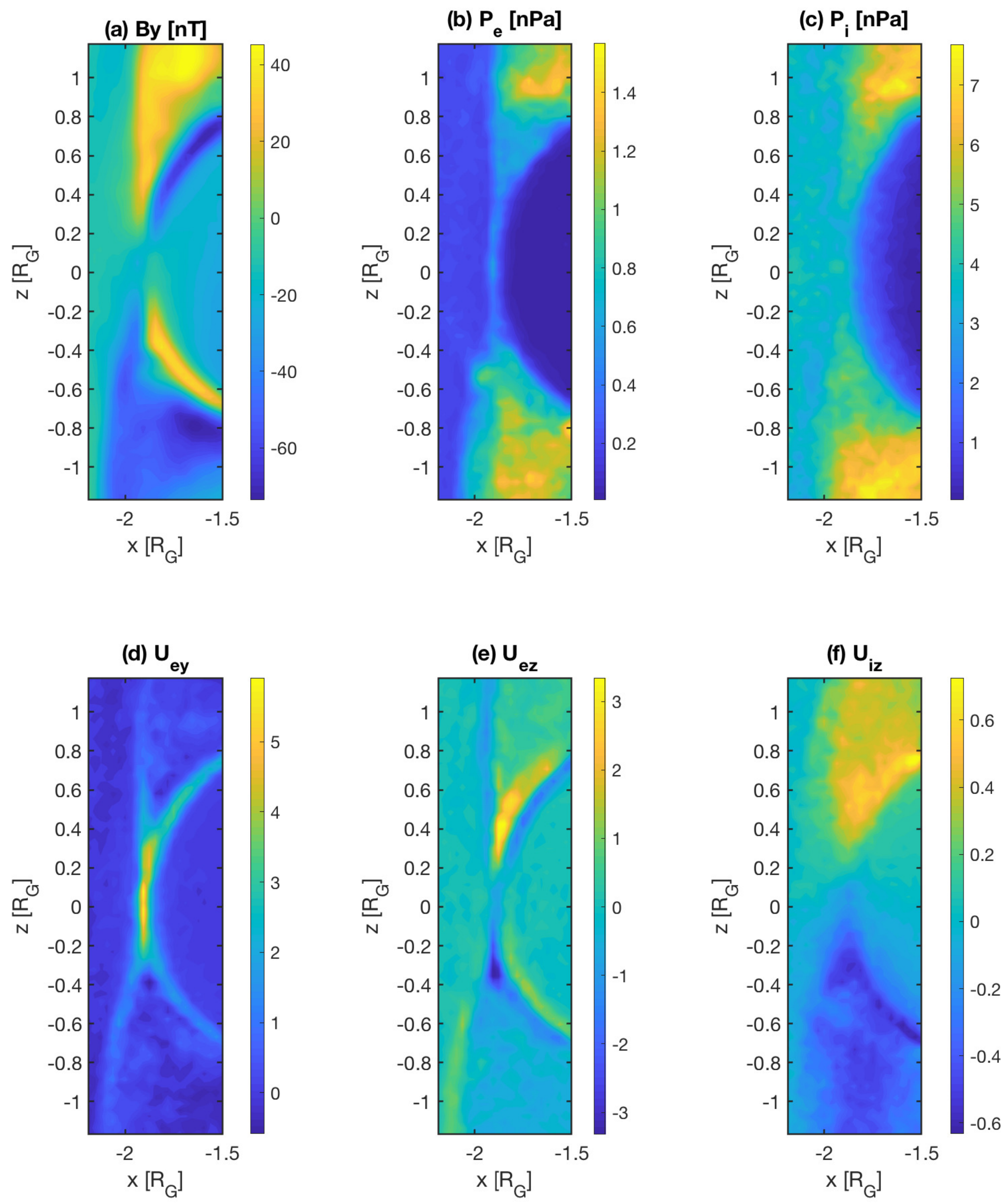

This article is protected by copyright. All rights reserved. 


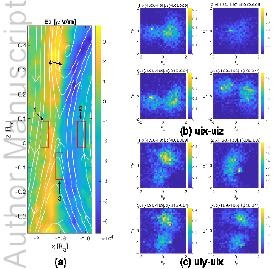


Figure 12.
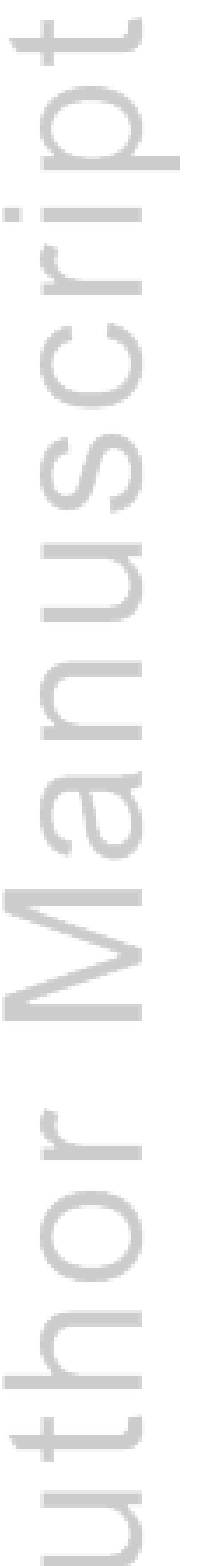

This article is protected by copyright. All rights reserved. 


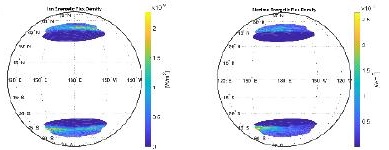




\section{(3) $100 R_{G}$}

\section{$174 R_{G}$}
(2) $1.0 \mathrm{R}_{\mathrm{G}}$

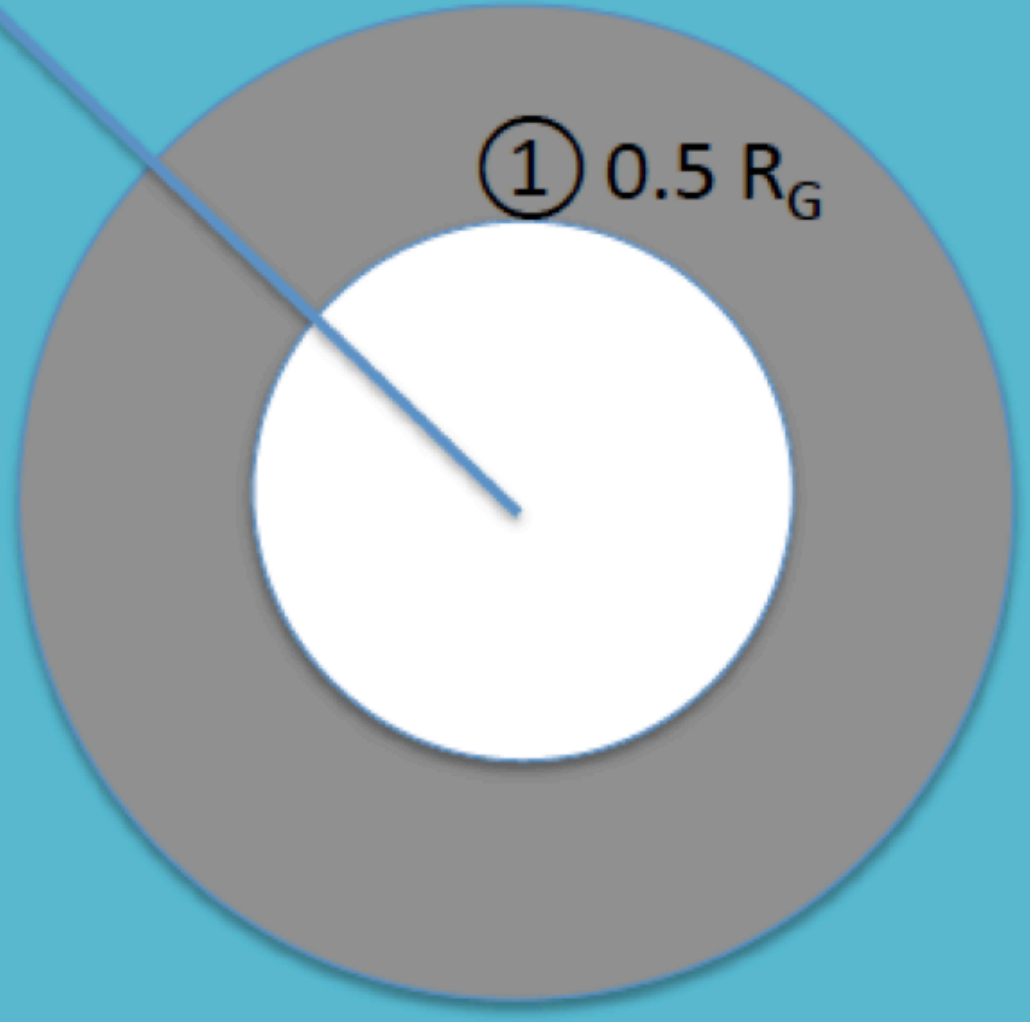

2019JA026643-f02-z-.png

This article is protected by copyright. All rights reserved. 


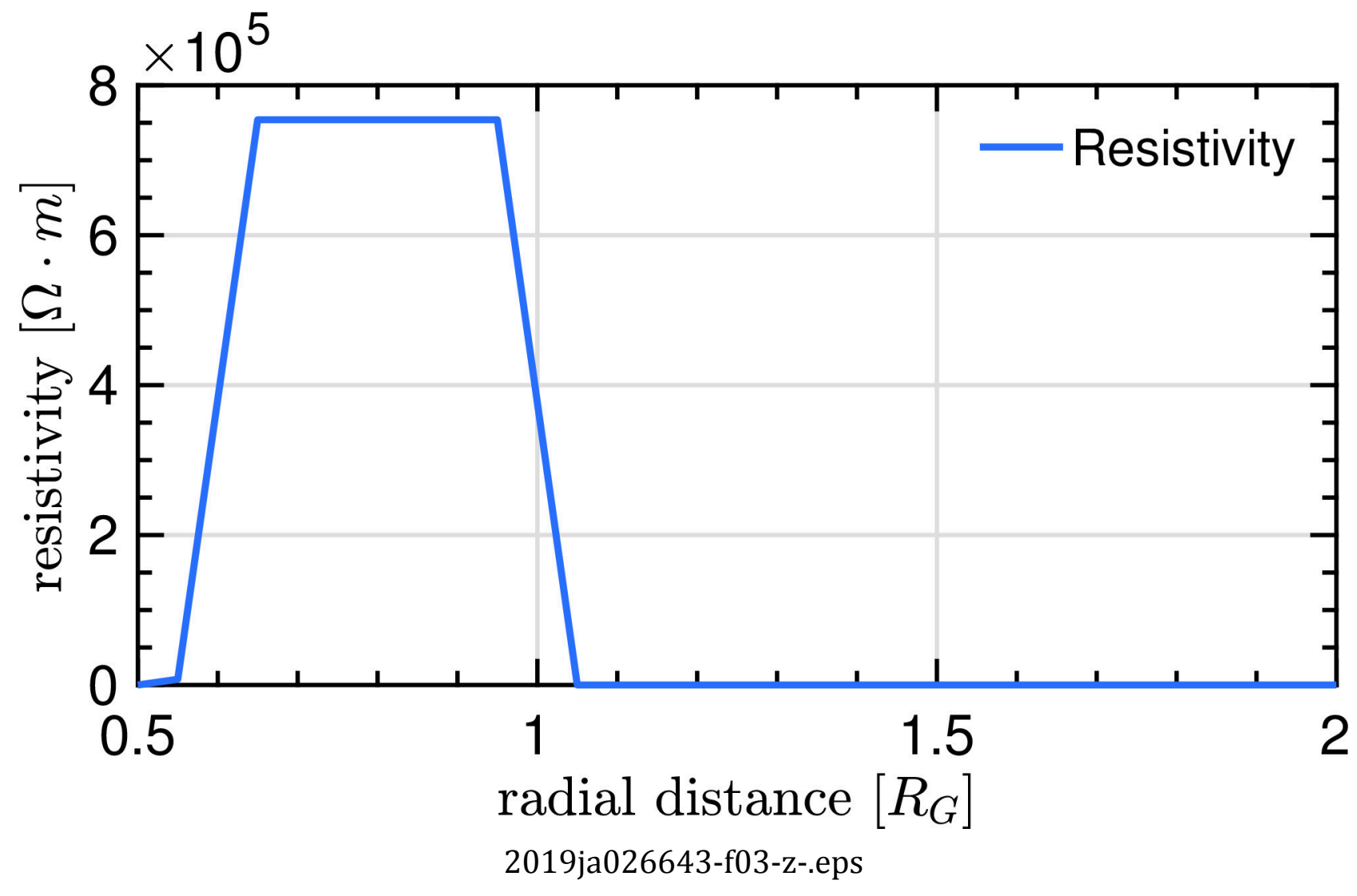

This article is protected by copyright. All rights reserved. 


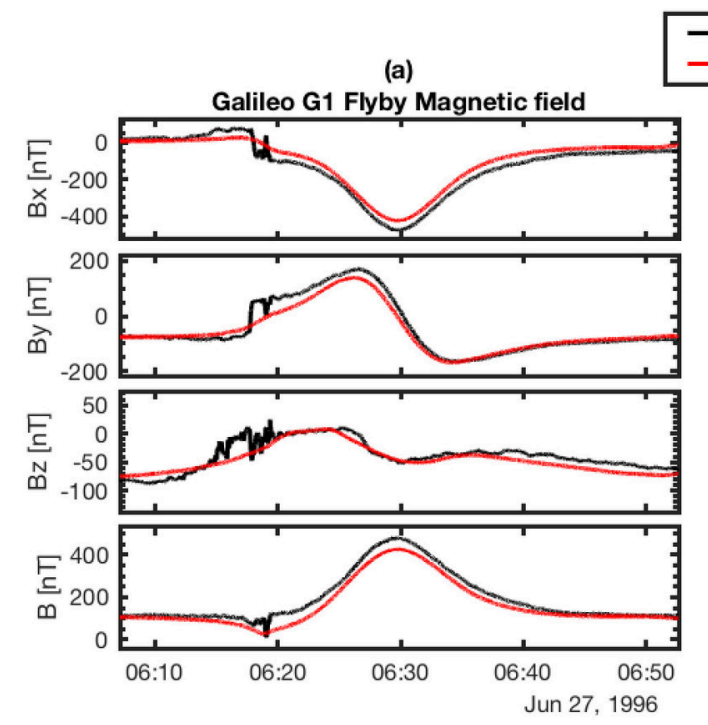

(b)

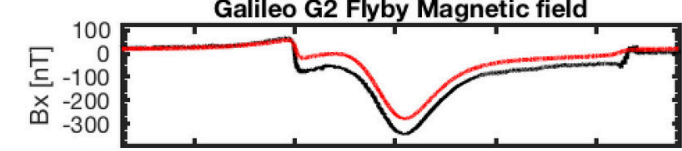

(c)

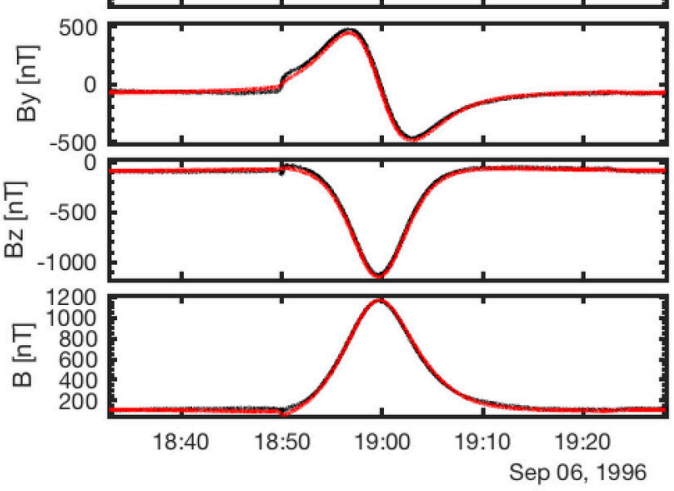

(d)
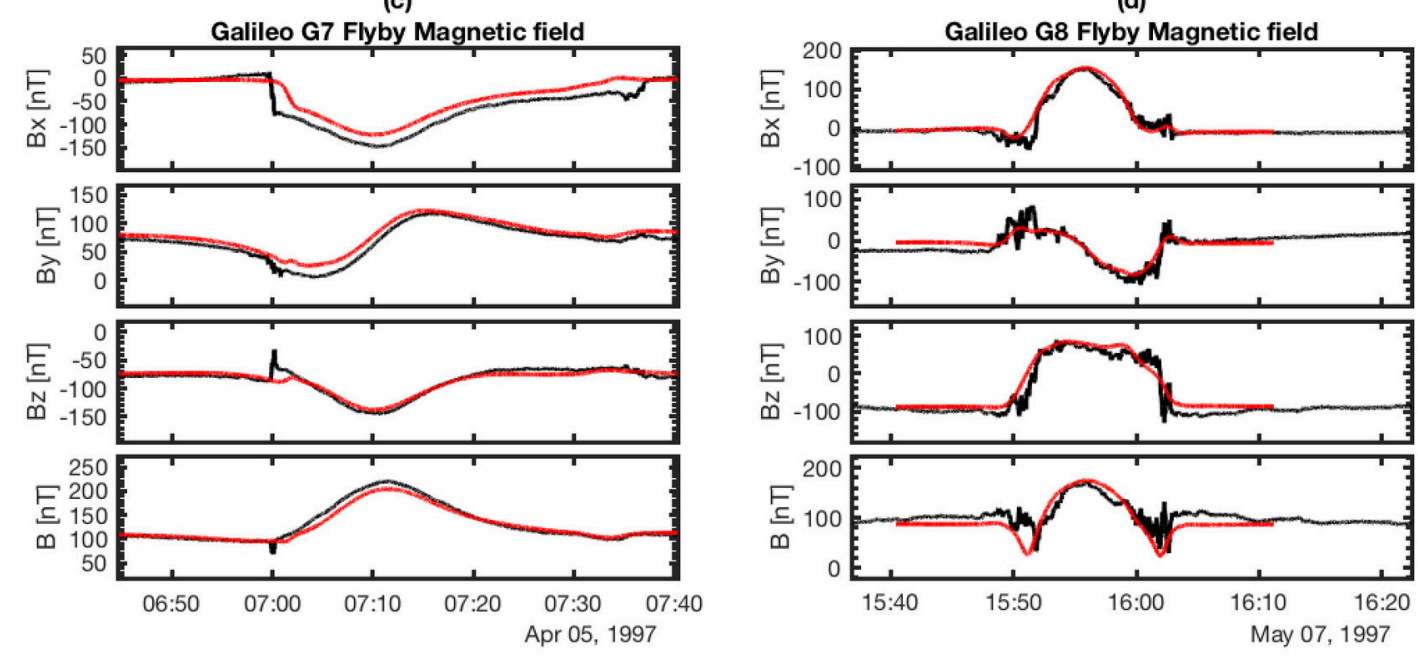

(e)

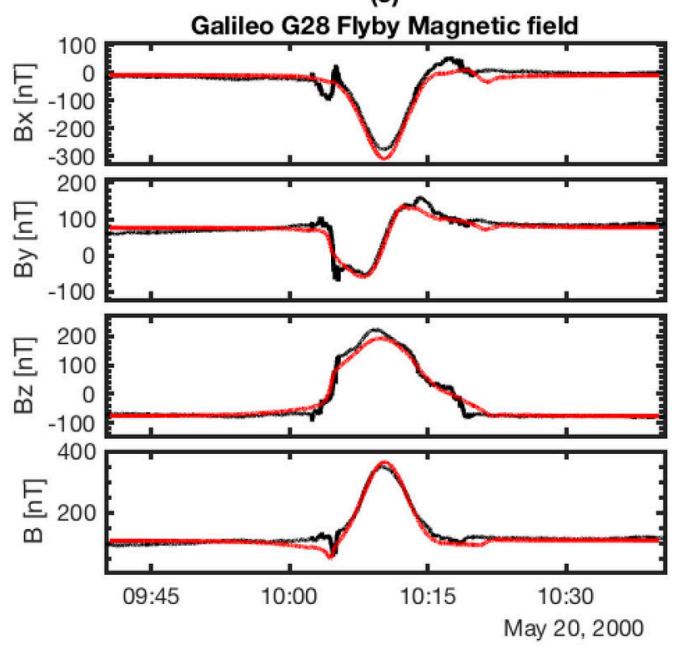

(f)
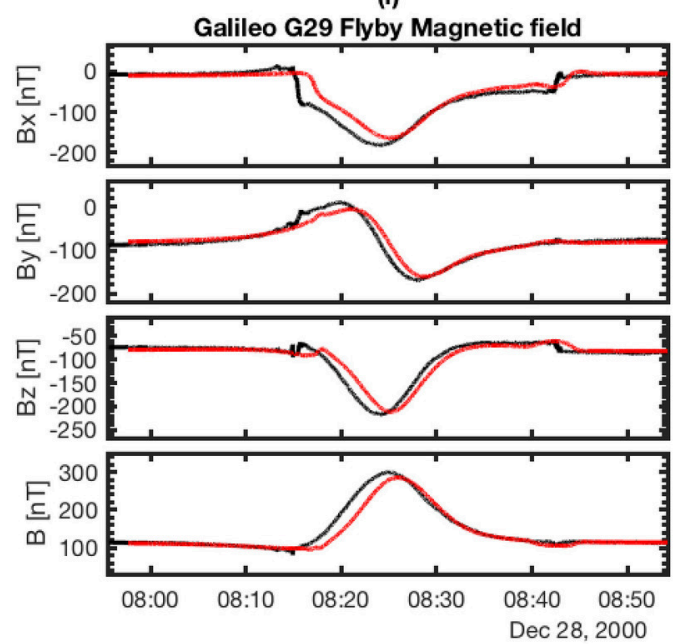

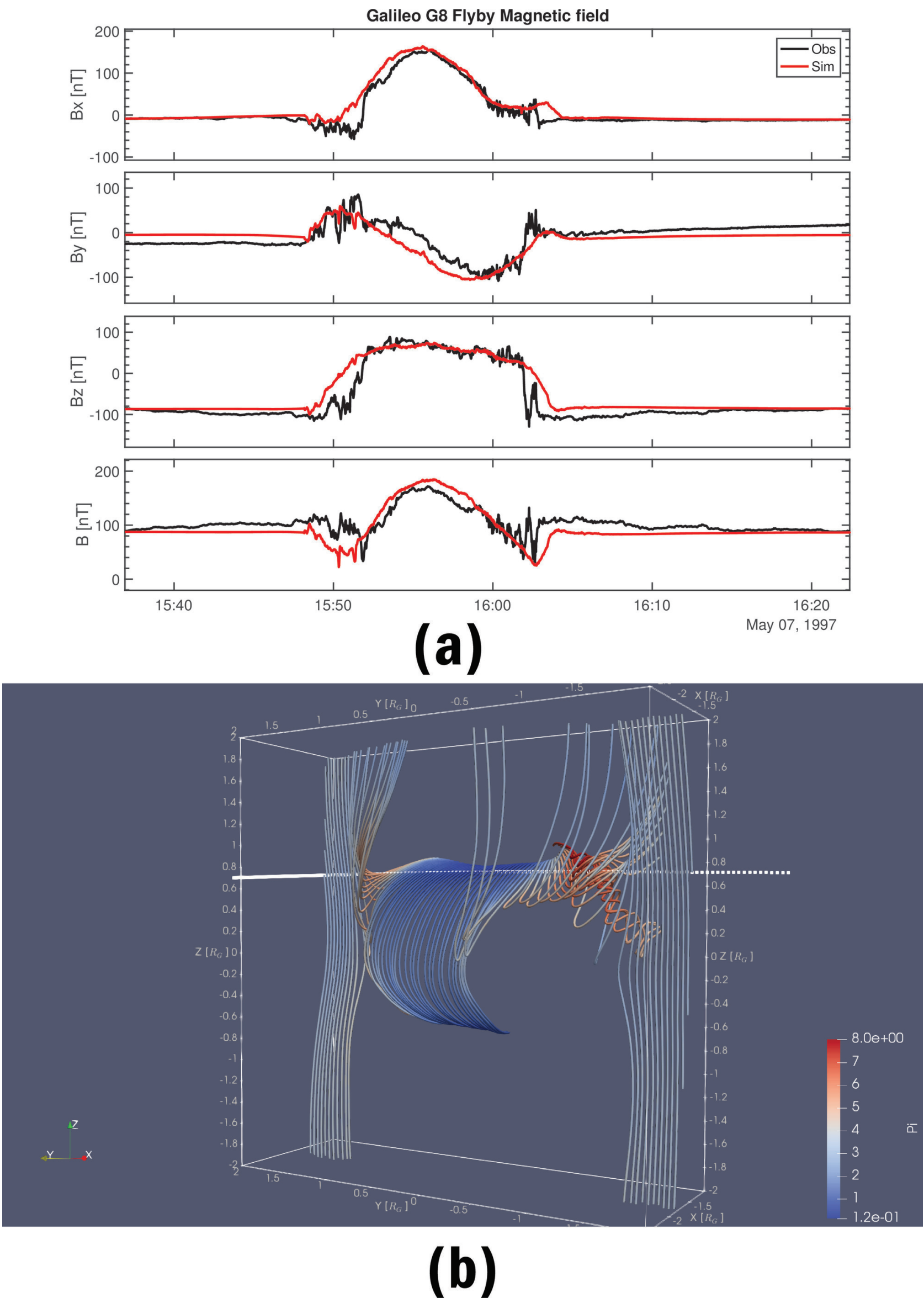

2019ja026643-f07-z-.eps

This article is protected by copyright. All rights reserved. 

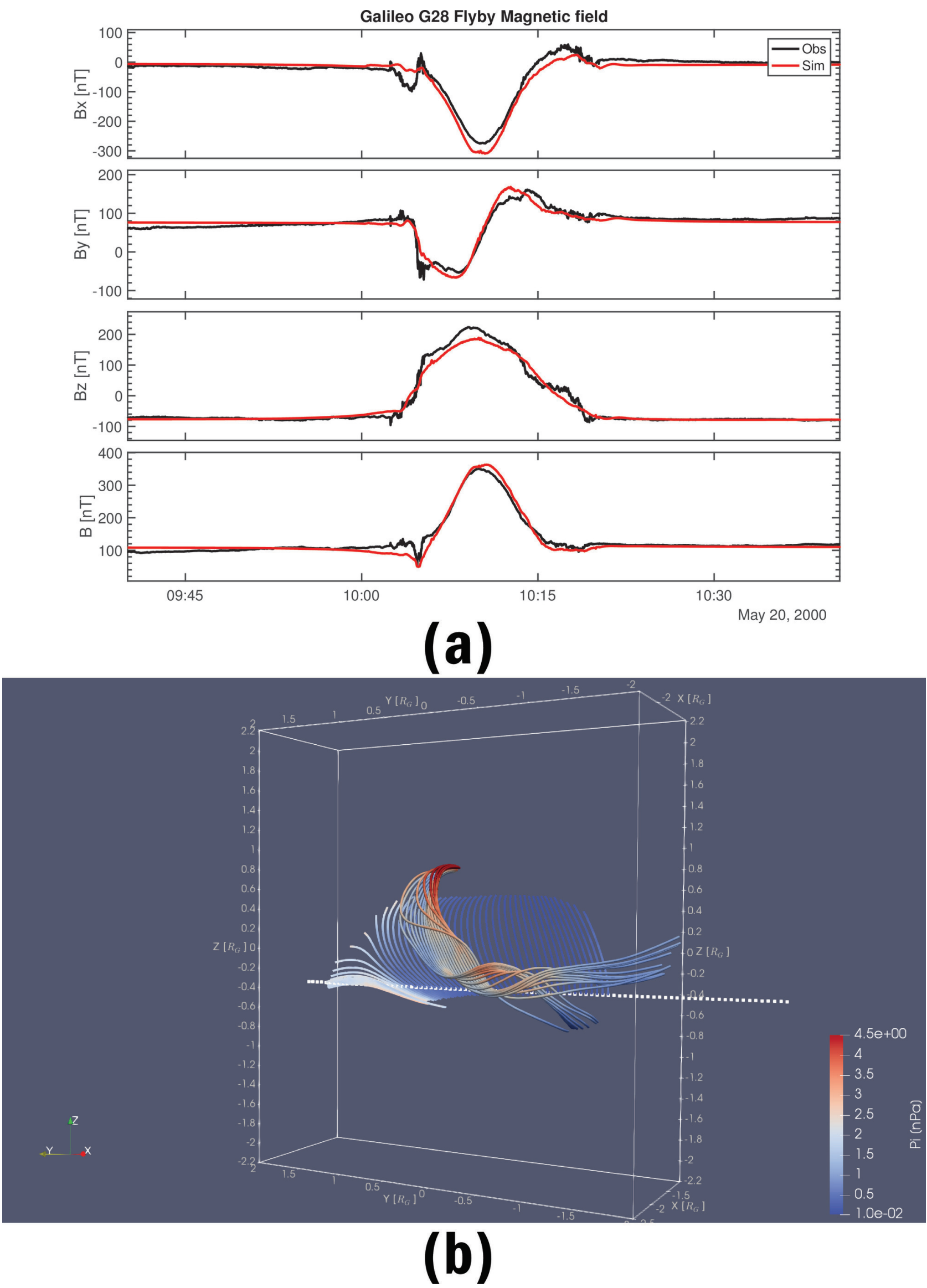

2019ja026643-f08-z-.eps

This article is protected by copyright. All rights reserved. 

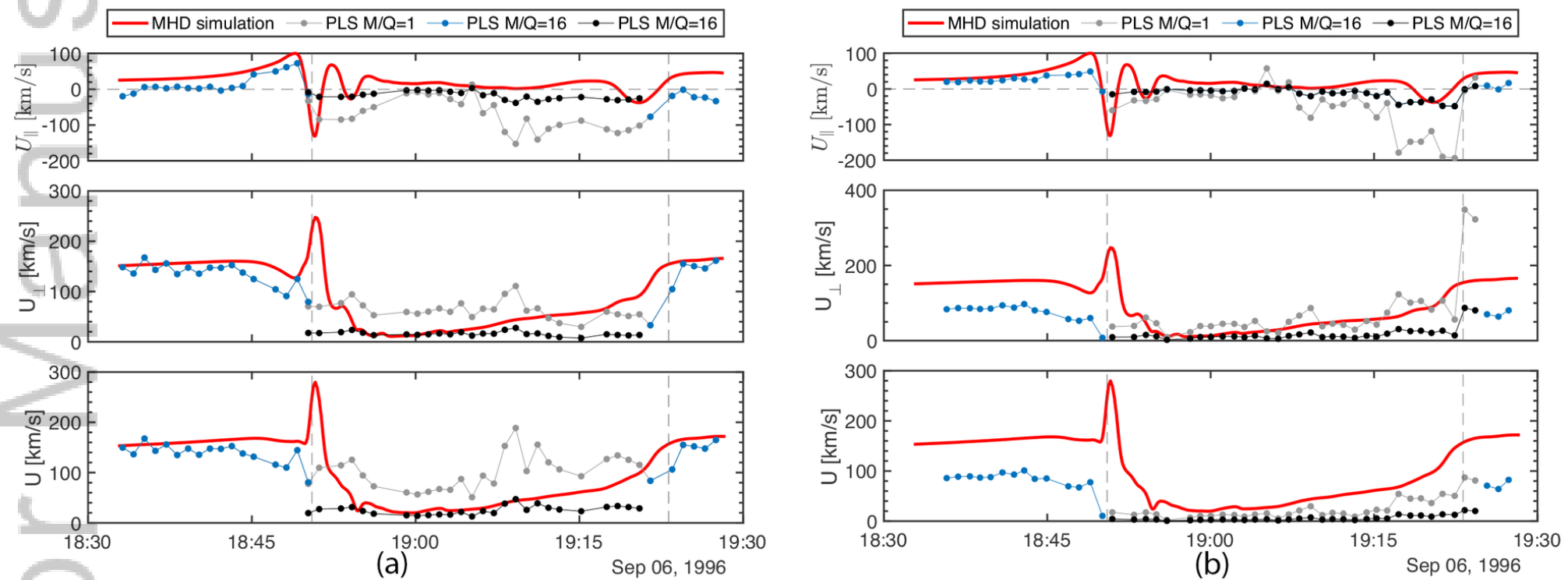

2019JA026643-f09-z-.png

This article is protected by copyright. All rights reserved. 
(a) By [nT]

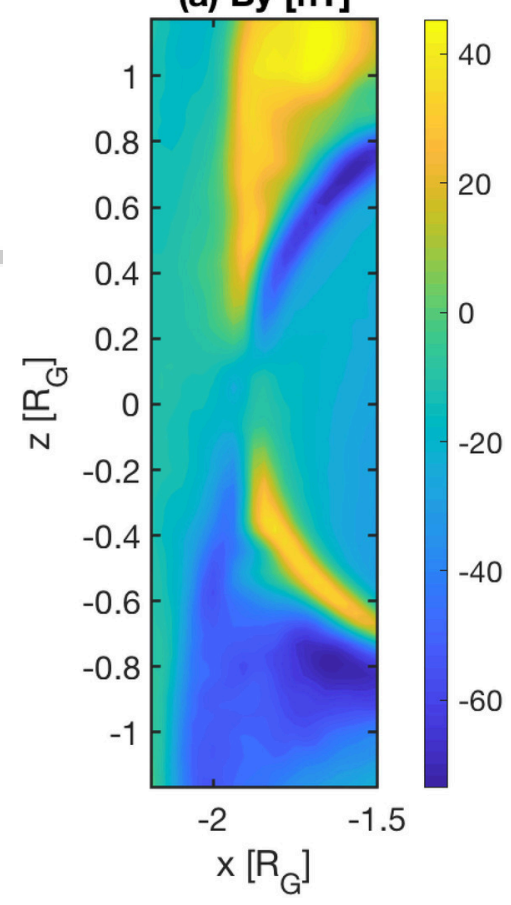

(d) $U_{\text {ey }}$

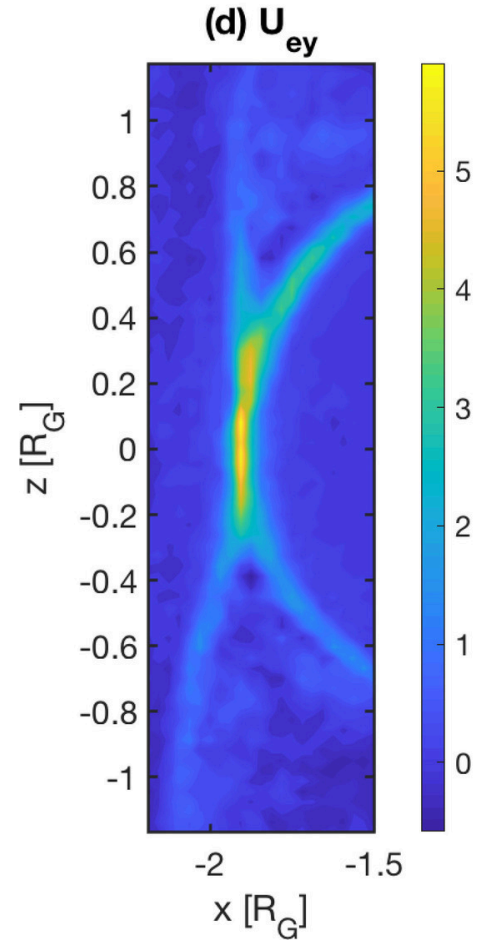

(b) $\mathrm{P}_{\mathrm{e}}[\mathrm{nPa}]$

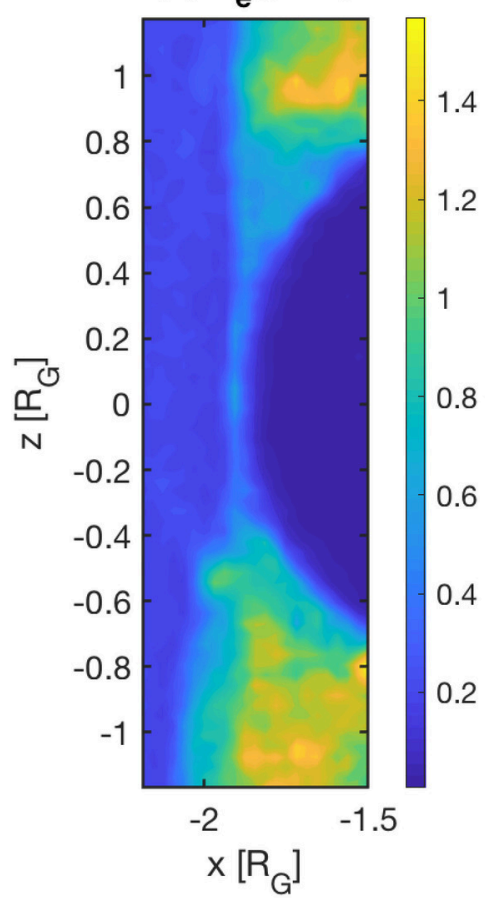

(e) $U_{e z}$

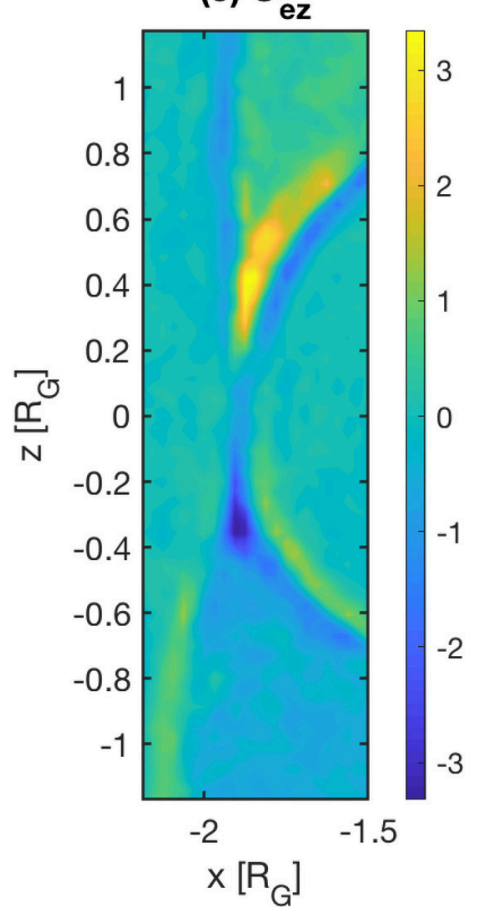

2019ja026643-f10-z-.eps (c) $\mathrm{P}_{\mathrm{i}}$ [nPa]
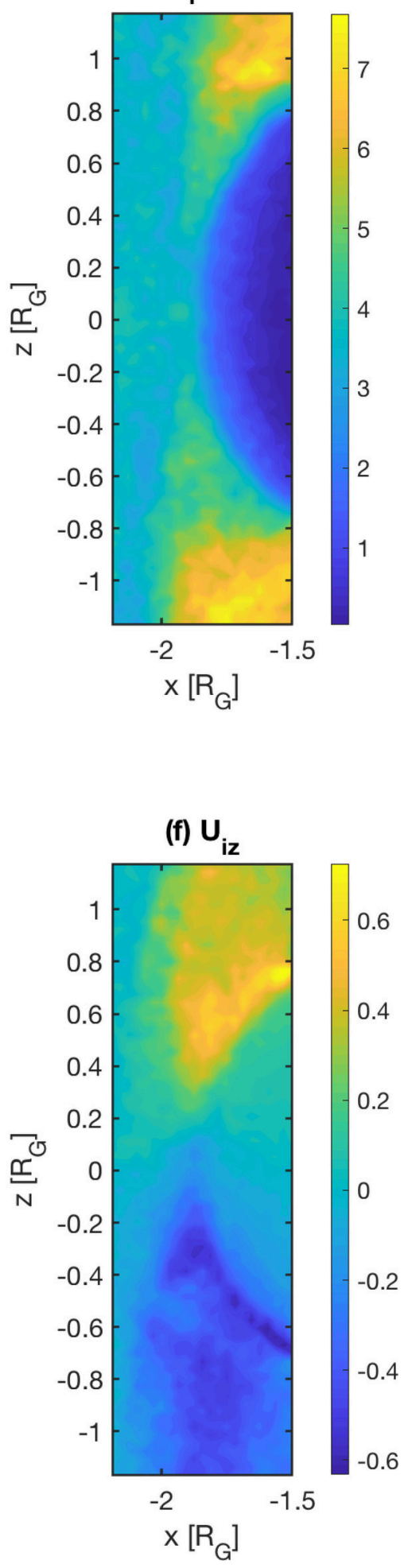

This article is protected by copyright. All rights reserved. 


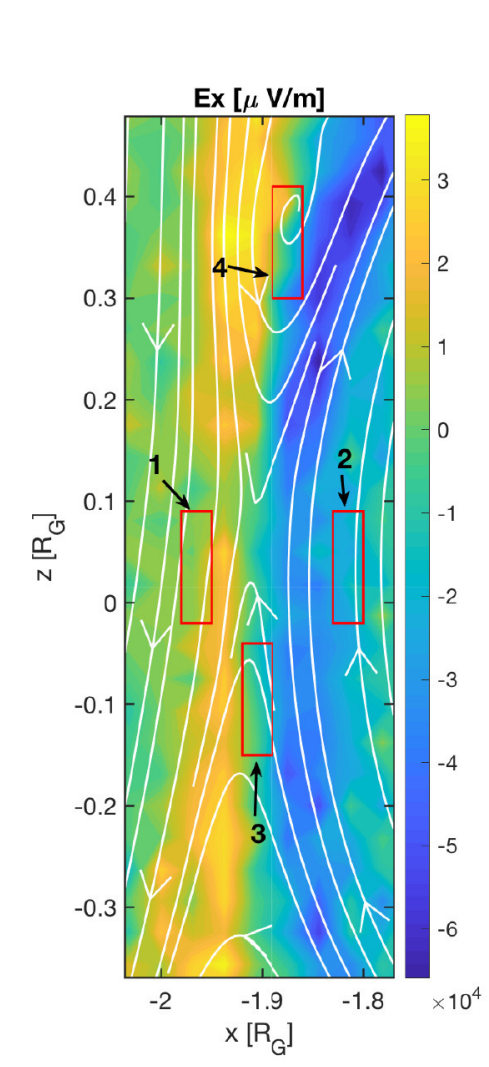

(a)
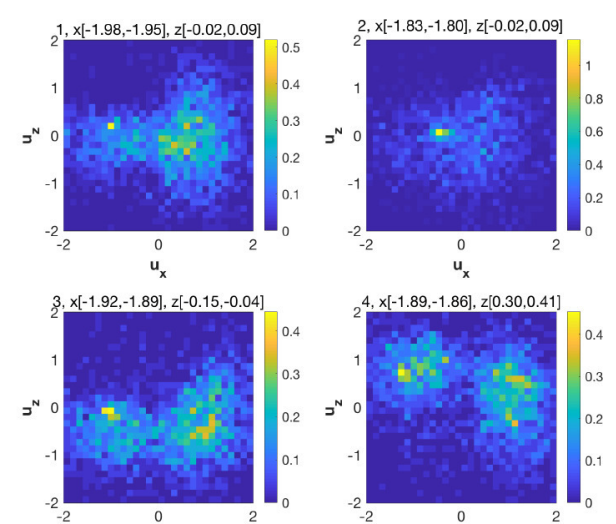

(b) uix-uiz
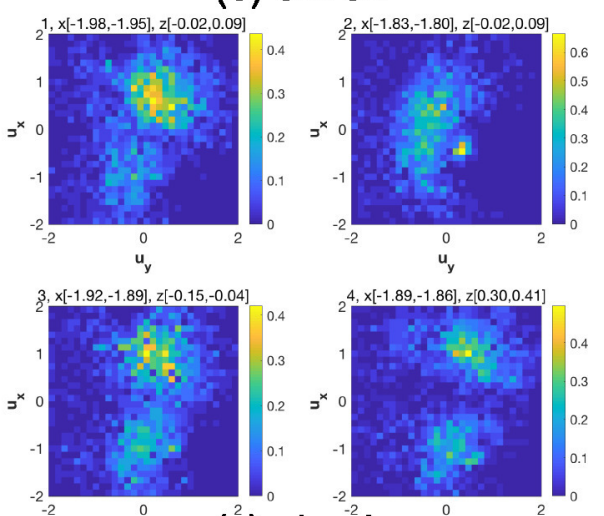

(c) uiy-uix $\quad 0^{2}$

2019ja026643-f11-z-.eps 


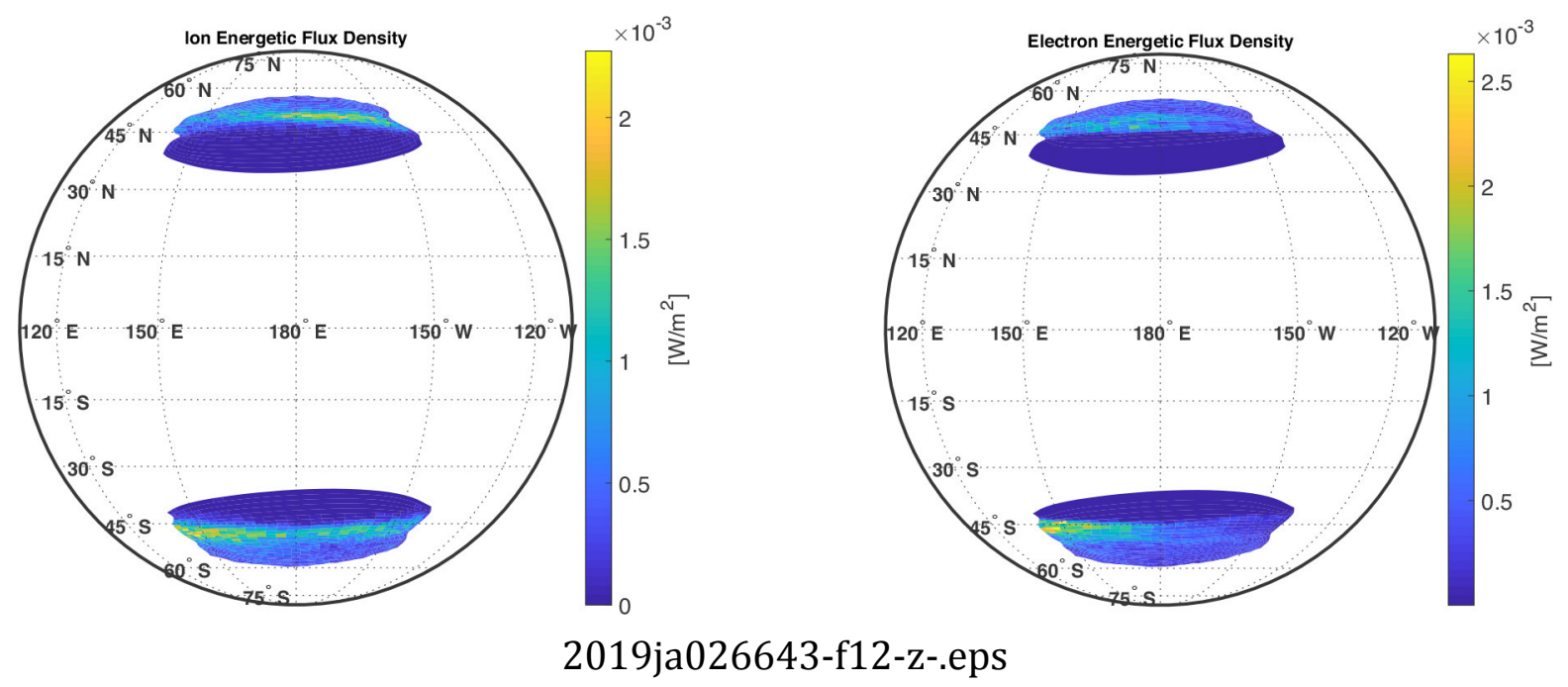

This article is protected by copyright. All rights reserved. 